(c) 2000 International Press

Adv. Theor. Math. Phys. 4 (2000) 571-615

\title{
Aspects of the conformal
}

\section{Operator Product Expansion}

\section{in AdS/CFT Correspondence}

Laurent Hoffmann, Anastasios C. Petkou and Werner Rühl

\author{
Department of Physics, Theoretical Physics \\ University of Kaiserslautern, Postfach 3049 \\ 67653 Kaiserslautern, Germany \\ hoffmann@physik.uni-kl.de \\ petkou@physik.uni-kl.de \\ ruehl@physik.uni-kl.de
}

\begin{abstract}
We present a detailed analysis of a scalar conformal fourpoint function obtained from AdS/CFT correspondence. We study the scalar exchange graphs on $\mathrm{AdS}_{d+1}$ and discuss their analytic properties. Using methods of conformal partial wave analysis, we present a general procedure to study conformal
\end{abstract}


four-point functions in terms of exchanges of scalar and tensor fields. The logarithmic terms in the four-point function are connected to the anomalous dimensions of the exchanged fields. Comparison of the results from $\mathrm{AdS}_{d+1}$ graphs with the conformal partial wave analysis suggests a possible general form for the operator product expansion of scalar fields in the boundary $\mathrm{CFT}_{d}$.

\section{Introduction}

The duality between string or $M$-theory compactifications on $\operatorname{AdS}_{d+1}$ and $d$-dimensional superconformal gauge theories suggested by AdS/CFT correspondence [1] has been the subject of intensive research over the past couple of years (for a recent review see [2]). Gradually, the emerging picture takes the form of the long-sought string/gauge theory relationship [3]. Recently, in a minkowsian version of the correspondence the $d$-dimensional conformal field theory (CFT) has been discussed in the context of local quantum field theory [4] defined on a standard (flat) compactified Minkowski space $M_{1, d-1}^{c}$. This space arises as the boundary of the $\mathrm{AdS}_{1, d}$ space-time. The isometry group of both spaces is $S O(d, 2)$ and the state space of the boundary CFT is related to the state space of the bulk theory [5].

Such a view of the AdS/CFT correspondence implies that the known local structure of conformal field theory, (see for example [6] and references therein), is connected to the local structure of the the field (or string) theory living on AdS. In particular, harmonic analysis on the isometry group $S O(d, 2)$ ("conformal partial wave analysis" CPWA), of $n$-point functions of the boundary CFT should be valid. This is equivalent to the existence of an operator product expansion (OPE) for the boundary CFT. Such expansions are convergent in a topology 
defined by the $n$-point functions on which they are applied (CPWA), or into which they are inserted (OPE). Perhaps the most well-known application ground for CPWA and OPEs is the (Euclidean) case $d=4$, when the boundary CFT is the $\mathcal{N}=4 \mathrm{SYM}$ theory with gauge group $S U(N)$. In that case, the large- $N$, large- $\lambda$ expansion $\left(\lambda=g_{Y M}^{2} N\right.$ with $g_{Y M}$ being the gauge coupling), corresponds to a perturbative form of the AdS theory in terms of the so-called "Witten graphs" [1]. Technical exploitations of the AdS/CFT correspondence are mainly based on this graphical expansion $[8,9]$.

Our aim in this work is to make a thorough investigation of a fourpoint function of scalar fields in the boundary CFT obtained from a graphical expansion in AdS. We choose to work in general dimensions $d$ to ensure a broad applicability of our results. In Section 2 we set the stage for our study by considering a theory on AdS with a single cubic local interaction term. This may be viewed as the minimal AdS theory leading to a non-trivial four-point function in the boundary. Locality arguments applied to the boundary CFT require the analyticity of the AdS calculations. In Section 3 we present the results of the AdS calculations in the direct and the crossed channels. The direct channel poses no analyticity problems. Complications arise in the crossed channels where non-analytic terms might arise. We prove that the possible non-analytic terms drop out by virtue of highly non-trivial identities for generalized hypergeometric functions, thus demonstrating that the corresponding CFT amplitudes admit an OPE. We present a systematic harmonic analysis of CFT four-point functions in Section 4 based on conformal exchange graphs for scalar and tensor fields. These graphs enable us to obtain the general contribution of scalar and tensor fields in a four-point function when the OPE is inserted in the direct channel. An important point of our analysis is the interpretation of the logarithmic terms which appear in four-point function calculations in terms of 
the anomalous dimensions of the exchanged scalar and tensor fields. This interpretation is by no means new as it has already been used by Symanzik [10] and two of the present authors [11, 13]. In Section 5 we combine the AdS results with the direct channel OPE. This allows the recursive determination of the anomalous dimensions and couplings of all scalar and tensor fields in the OPE. Our results seem to draw a clear picture for the OPE of two scalar fields of the boundary CFT; it contains a) the full contribution from the boundary conformal field which corresponds to the AdS-field in the cubic bulk interaction ${ }^{1}$ and b) infinite towers of conformal scalar and tensor fields whose canonical dimensions (e.g., the part of the dimension which does not depend on the coupling), and tensor rank are simply related to the dimensions of the external fields in the OPE. Finally, we summarize our results and comment on possible extensions of our program in Section 6 .

\section{General remarks}

It is well-known that CFT determines the form of two- and three-point functions of general tensor fields up to constants (for a review see [14]). Although these constants capture in general non-trivial dynamical effects, four-point functions are the minimal ones whose functional form depends in an essential way on the dynamics. From conformal invariance four-point functions are determined only up to a general analytic function of two variables. Namely, consider the four-point function

$$
\left\langle\mathcal{O}_{1}\left(x_{1}\right) \mathcal{O}_{3}\left(x_{3}\right) \mathcal{O}_{2}\left(x_{2}\right) \mathcal{O}_{4}\left(x_{4}\right)\right\rangle
$$

where the scalar fields $\mathcal{O}_{i}\left(x_{i}\right), i=1, \ldots, 4$ have dimensions $\Delta_{i}$ respectively. Denoting $x_{i j}=x_{i}-x_{j}$ the four-point function (1) can be

\footnotetext{
${ }^{1}$ Our results differ in this point from the recent claims in [15].
} 
expanded in powers of $x_{i j}^{2}$, determined solely from $\Delta_{i}$, and an analytic function $F(u, v)$ of the two biharmonic ratios

$$
u=\frac{x_{13}^{2} x_{24}^{2}}{x_{12}^{2} x_{34}^{2}}, \quad v=\frac{x_{14}^{2} x_{23}^{2}}{x_{12}^{2} x_{34}^{2}}
$$

There exists then a physical real analyticity domain for $F(u, v)$ with [11]

$$
|1+u-v| \leq 2 u^{\frac{1}{2}}, \quad|1+v-u| \leq 2 v^{\frac{1}{2}}
$$

and a possible expansion is

$$
F(u, v)=\sum_{n, m=0}^{\infty} \frac{u^{n}(1-v)^{m}}{n ! m !} A_{n m}\left(\Delta_{i} ; d\right)
$$

An expansion such as (4) is useful in the direct channel limit

$$
x_{1} \rightarrow x_{3}, \quad x_{2} \rightarrow x_{4}, \quad(u \rightarrow 0, v \rightarrow 1),
$$

and it is obtained from the calculation of conformal graphs $[11,12$, $13,16,17]$. In almost all four-point function calculations one relies on a perturbative expansion in some small parameter e.g. a coupling constant or $1 / N$. Such a perturbative expansion implies the dependence of conformal dimensions on the coupling constants (or $1 / N$ ) and gives, for a specific graph $\Gamma$, an expansion of the form

$$
F_{\Gamma}(u, v)=\sum_{n, m=0}^{\infty} \frac{u^{n}(1-v)^{m}}{n ! m !}\left[\sum_{k=0}^{\mathcal{K}} A_{n m}^{(k)}\left(\Delta_{i} ; d\right)(\ln u)^{k}\right],
$$

where $\mathcal{K}$ depends on the perturbative order. One important point here is the appearance of the logarithmic terms on the r.h.s. of (6). These terms are not involved in the discussion of the analytic properties of the expansion (6) as they can in principle be summed up and exponentiated giving just an "anomalous" contribution to the dimensions of the exchanged fields $[10,11,12,13]$. Logarithmic terms frequently occur in 
conformal $n$-point functions and are in fact necessary in order to ensure the correct conformal properties when the external points come close together $[14,18]$.

In the context of AdS/CFT correspondence [1] one is equipped with a standard procedure to generate a perturbative expansion for conformal four-point functions. Namely, the relation of a (local) field theory on AdS to a CFT on the boundary can be schematically written as

$$
Z_{\mathrm{AdS}} \equiv \int(\mathcal{D} \phi) e^{-S[\phi]} \rightarrow Z\left[\phi_{0}\right] \equiv\left\langle e^{\int \mathrm{d}^{d} x \phi_{0}(x) \mathcal{O}_{\phi}(x)}\right\rangle=e^{W_{\mathrm{CFT}}\left[\phi_{0}\right]}
$$

where $W_{\mathrm{CFT}}\left[\phi_{0}\right]$ is the generating functional for connected $n$-point functions of the field $\mathcal{O}_{\phi}(x)$ in the boundary CFT, when $\phi_{0}(x)$ plays the role of an external source. Passing from $Z$ to $Z\left[\phi_{0}\right]$ involves solving the classical field equations for the AdS field ${ }^{2} \phi\left(x_{0}, x\right)$ with boundary conditions such that $\left.\phi\left(x_{0}, x\right)\right|_{\text {addS }}=\phi_{0}(x)$. When the AdS action involves non-quadratic terms it can only be evaluated within a perturbative expansion in the coupling constant. Such an expansion has a definite interpretation in terms of "Witten graphs" $[1,8,9]$ connecting points in the boundary of AdS via interactions taking place in the bulk. The result is a perturbative expansion for the $n$-point functions of the boundary CFT.

Here we consider the simplest local field theory on AdS which gives rise to non-trivial four-point functions of the boundary CFT. Namely,

\footnotetext{
${ }^{2}$ We consider the Euclidean version of $\mathrm{AdS}_{d+1}$ space where $\mathrm{d} \hat{x}^{\mu} \mathrm{d} \hat{x}_{\mu}=$ $\frac{1}{x_{0}^{2}}\left(\mathrm{~d} x_{0} \mathrm{~d} x_{0}+\mathrm{d} x^{i} \mathrm{~d} x^{i}\right)$, with $i=1, . ., d$. and $\hat{x}_{\mu}=\left(x_{0}, x_{i}\right)$. The boundary of this space is isomorphic to $\mathbf{S}^{\mathrm{d}}$ since it consists of $\mathbf{R}^{\mathrm{d}}$ at $x_{0}=0$ and a single point at $x_{0}=\infty$.
} 
we consider the following action for the AdS scalar fields $\phi(\hat{x})$ and $\sigma(\hat{x})$

$$
\begin{aligned}
S=\int & \mathrm{d}^{d+1} \hat{x} \sqrt{g}\left(\frac{1}{2} \partial_{\mu} \phi(\hat{x}) \partial^{\mu} \phi(\hat{x})+\frac{1}{2} \tilde{m}^{2} \phi^{2}(\hat{x})\right. \\
& \left.+\frac{1}{2} \partial_{\mu} \sigma(\hat{x}) \partial^{\mu} \sigma(\hat{x})+\frac{1}{2} m^{2} \sigma^{2}(\hat{x})+\frac{\gamma_{*}}{2} \phi^{2}(\hat{x}) \sigma(\hat{x})\right) .
\end{aligned}
$$

The action (8) gives rise to a boundary CFT of the scalar fields $\mathcal{O}_{\phi}(x)$ and $\mathcal{O}_{\sigma}(x)$ with corresponding two-point functions $[8,9]$

$$
\begin{aligned}
\left\langle\mathcal{O}_{\phi}\left(x_{1}\right) \mathcal{O}_{\phi}\left(x_{2}\right)\right\rangle & =C_{\tilde{\Delta}} \frac{1}{x_{12}^{2 \tilde{\Delta}}}, \quad \tilde{\Delta}=\frac{d}{2}+\sqrt{\tilde{m}^{2}+\frac{d^{2}}{4}} \\
\left\langle\mathcal{O}_{\sigma}\left(x_{1}\right) \mathcal{O}_{\sigma}\left(x_{2}\right)\right\rangle & =C_{\Delta} \frac{1}{x_{12}^{2 \Delta}}, \quad \Delta=\frac{d}{2}+\sqrt{m^{2}+\frac{d^{2}}{4}} \\
C_{\Delta} & =\frac{2\left(\Delta-\frac{1}{2} d\right) \Gamma(\Delta)}{\pi^{\frac{1}{2} d} \Gamma\left(\Delta-\frac{1}{2} d\right)} .
\end{aligned}
$$

It also implies the existence of the three-point function $[8,16]$

$$
\begin{aligned}
\left\langle\mathcal{O}_{\phi}\left(x_{1}\right) \mathcal{O}_{\phi}\left(x_{2}\right) \mathcal{O}_{\sigma}\left(x_{3}\right)\right\rangle & =\gamma_{*} g_{\tilde{\Delta} \tilde{\Delta} \Delta} \frac{1}{\left(x_{12}^{2}\right)^{\tilde{\Delta}-\frac{1}{2} \Delta}\left(x_{13}^{2} x_{23}^{2}\right)^{\frac{1}{2} \Delta}} \\
g_{\tilde{\Delta} \tilde{\Delta} \Delta} & =\frac{1}{4 \pi^{d}} \frac{\Gamma^{2}\left(\frac{1}{2} \Delta\right) \Gamma\left(\tilde{\Delta}-\frac{1}{2} \Delta\right) \Gamma\left(\tilde{\Delta}+\frac{1}{2} \Delta-\frac{1}{2} d\right)}{\Gamma^{2}\left(\tilde{\Delta}-\frac{1}{2} d\right) \Gamma\left(\Delta-\frac{1}{2} d\right)} .
\end{aligned}
$$

The coupling constant $\gamma_{*}$ is the parameter which induces the non-trivial dynamics of the boundary CFT. In principle, one should have information regarding its magnitude before trying to make sense of the "Witten graph" expansion. This is the case when $\phi(\hat{x})$ and $\sigma(\hat{x})$ correspond to Kaluza-Klein modes of the compactified supergravity theory and $\gamma_{*}$ is determined by the standard reduction procedure $[19,20]$ to be $\gamma_{*} \sim O(1 / N)$, where $S U(N)$ is the gauge group of the boundary CFT ${ }^{3}$. In this case one is able to order the perturbative expansion of the action (8) according to the number of cubic vertices. For the purposes of

\footnotetext{
${ }^{3}$ Recently, the full $\mathrm{AdS}_{d+1}$ action for the Kaluza-Klein modes has been evaluated up to quartic couplings [21].
} 
our work it suffices to assume that $\gamma_{*}$ is also a "small" parameter when $\phi(x)$ and $\sigma(x)$ are general scalar fields, as our results do not depend in an essential way on its magnitude.
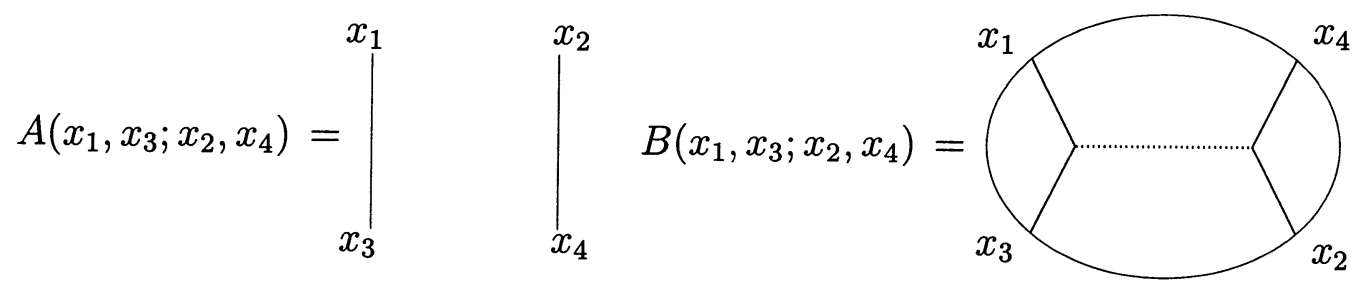

Figure 1: The $A$ and $B$ graphs. In the $A$ graph the solid lines correspond to the full $\mathcal{O}_{\phi}(x)$ propagator (9). In the $B$ graph, solid lines correspond to the "bulk-to-boundary" propagators (17) and the dotted line to the "bulk-to-bulk" one (18).

Our main interest is in the four-point function of the scalar field $\mathcal{O}_{\phi}(x)$. Up to tree "Witten graphs" standard AdS/CFT calculations give the following expansion

$$
\begin{aligned}
& \left\langle\mathcal{O}_{\phi}\left(x_{1}\right) \mathcal{O}_{\phi}\left(x_{3}\right) \mathcal{O}_{\phi}\left(x_{2}\right) \mathcal{O}_{\phi}\left(x_{4}\right)\right\rangle \\
& =A\left(x_{1}, x_{2} ; x_{3}, x_{4}\right)+A\left(x_{1}, x_{3} ; x_{2}, x_{4}\right)+A\left(x_{1}, x_{4} ; x_{3}, x_{2}\right) \\
& \quad+\gamma_{*}^{2}\left[B\left(x_{1}, x_{2} ; x_{3}, x_{4}\right)+B\left(x_{1}, x_{3} ; x_{2}, x_{4}\right)+B\left(x_{1}, x_{4} ; x_{3}, x_{2}\right)\right]
\end{aligned}
$$

where the $A$ and $B$ terms are depicted in Fig.1. Note that we consider the full four-point function and not only its connected part. The explicit expressions for the graphs $A$ and $B$ are given by

$$
\begin{aligned}
& A\left(x_{1}, x_{2} ; x_{3}, x_{4}\right)=C_{\tilde{\Delta}}^{2} \frac{1}{\left(x_{12}^{2} x_{34}^{2}\right)^{\tilde{\Delta}}} \\
& B\left(x_{1}, x_{2} ; x_{3}, x_{4}\right) \\
& =\int \frac{\mathrm{d}^{d+1} \hat{y} \mathrm{~d}^{d+1} \hat{z}}{y_{0}^{d+1} z_{0}^{d+1}} K_{\tilde{\Delta}}\left(x_{1}, \hat{y}\right) K_{\tilde{\Delta}}\left(x_{2}, \hat{y}\right) G_{\Delta}(\hat{y}, \hat{z}) K_{\tilde{\Delta}}\left(x_{3}, \hat{z}\right) K_{\tilde{\Delta}}\left(x_{4}, \hat{z}\right)
\end{aligned}
$$

and the standard forms of the "bulk-to-boundary" and "bulk-to-bulk" 
propagators that we use are $[16,17]$

$$
\begin{aligned}
K_{\Delta}(x, \hat{y}) & =k_{\Delta}\left[\frac{x_{0}}{x_{0}^{2}+(x-y)^{2}}\right]^{\Delta}, \quad k_{\Delta}=\frac{\Gamma(\Delta)}{\pi^{\frac{1}{2} d} \Gamma\left(\Delta-\frac{1}{2} d\right)}, \\
G_{\Delta}(x, y) & =\mathcal{G}_{\Delta} q^{-\Delta}{ }_{2} F_{1}\left(\frac{1}{2}(\Delta+1), \frac{1}{2} \Delta ; \Delta-\frac{d}{2}+1 ; q^{-2}\right) \\
\mathcal{G}_{\Delta} & =\frac{\Gamma(\Delta)}{2^{\Delta+1} \pi^{\frac{1}{2} d} \Gamma\left(\Delta-\frac{1}{2} d+1\right)}, \quad q^{2}=\frac{x_{0}^{2}+y_{0}^{2}+(x-y)^{2}}{2 x_{0} y_{0}}
\end{aligned}
$$

The disconnected $A$ graphs simply give

$$
\frac{C_{\tilde{\Delta}}^{2}}{\left(x_{13}^{2} x_{24}^{2}\right)^{\tilde{\Delta}}}+\frac{C_{\tilde{\Delta}}^{2}}{\left(x_{12}^{2} x_{34}^{2}\right)^{\tilde{\Delta}}}\left[1+v^{-\tilde{\Delta}}\right] .
$$

The integrations in the "exchange graphs" $B$ can be done using either Symanzik's method [22] or following [17] (see Appendix A), when one obtains the following convenient representation as a Mellin-Barnes integral

$$
\begin{aligned}
& B\left(x_{1}, x_{3} ; x_{2}, x_{4}\right) \\
& =\frac{\kappa}{\left(x_{12}^{2} x_{34}^{2}\right)^{\tilde{\Delta}}} \int_{\mathcal{C}} \frac{\mathrm{d} s}{2 \pi \mathrm{i}} \Gamma^{2}(-s) \\
& \cdot\left[\frac{\Gamma^{4}(\tilde{\Delta}+s) \Gamma^{2}\left(\frac{1}{2} \Delta+\tilde{\Delta}-\frac{1}{2} d\right) \Gamma\left(\frac{1}{2} \Delta-\tilde{\Delta}-s\right)}{\Gamma(2 \tilde{\Delta}+2 s) \Gamma\left(\Delta-\frac{1}{2} d+1\right) \Gamma\left(\frac{1}{2} \Delta+\tilde{\Delta}-\frac{1}{2} d-s\right)}\right. \\
& \quad \times{ }_{3} F_{2}\left(\frac{1}{2} \Delta+\tilde{\Delta}-\frac{1}{2} d, \frac{1}{2} \Delta+\tilde{\Delta}-\frac{1}{2} d, \frac{1}{2} \Delta-\tilde{\Delta}-s ;\right. \\
& \left.\Delta-\frac{1}{2} d+1, \tilde{\Delta}+\frac{1}{2} \Delta-\frac{1}{2} d-s ; 1\right) \\
& \left.\times u^{s}{ }_{2} F_{1}(\tilde{\Delta}+s, \tilde{\Delta}+s ; 2 \tilde{\Delta}+2 s ; 1-v)\right]
\end{aligned}
$$

where

$$
\kappa=\frac{\Gamma\left(2 \tilde{\Delta}-\frac{1}{2} d\right)}{8 \pi^{3 d / 2} \Gamma^{4}\left(\tilde{\Delta}-\frac{1}{2} d\right)} .
$$


The remaining two crossing symmetric $B$ graphs are obtained from (21) by suitable interchanges of the $x$ 's. Our strategy is now to present an explicit expression for the four-point function (14) and study its analyticity properties. This expression will then be compared with the CFT expectation which is simply the CPWA of the four-point function (14). The result will suggest the local form for the OPE of the conformal scalar field $\mathcal{O}_{\phi}(x)$ with itself.

\section{Explicit results for the AdS exchange graphs}

In this section we present the results for the AdS "exchange graphs" and discuss their analyticity properties.

\subsection{The direct channel}

Our starting point is the Mellin-Barnes representation (21) for the $B$ terms in (14). It is easy to see that (21) is suitable for studying the direct channel limit (5). The result of the integration is a double series expansion coming from the summation over the $\Gamma$-function poles included in the contour $\mathcal{C}$. It can be written down as (see Appendix A for the details)

$$
\begin{aligned}
& B\left(x_{1}, x_{3} ; x_{2}, x_{4}\right) \\
& =\frac{1}{\left(x_{12}^{2} x_{34}^{2}\right)^{\tilde{\Delta}}} \sum_{m, n=0}^{\infty} \frac{u^{n}(1-v)^{m}}{n ! m !}\left[-a_{n m}^{(1)} \ln u+b_{n m}^{(1)}+u^{\frac{1}{2} \Delta-\tilde{\Delta}} c_{n m}^{(1)}\right],
\end{aligned}
$$


where

$$
\begin{aligned}
& a_{n m}^{(1)}=- \kappa \frac{\Gamma^{2}(\tilde{\Delta}+n) \Gamma^{2}(\tilde{\Delta}+n+m)}{(n+1) ! \Gamma(2 \tilde{\Delta}+2 n+m)} \frac{1}{\left(\frac{1}{2} \Delta+\tilde{\Delta}-\frac{1}{2} d\right)} \\
& \times{ }_{3} F_{2}\left(\frac{1}{2} \Delta-\tilde{\Delta}+1, \frac{1}{2} \Delta+\tilde{\Delta}-\frac{1}{2} d+1+n, 1 ;\right. \\
&\left.n+2, \frac{1}{2} \Delta+\tilde{\Delta}-\frac{1}{2} d+1 ; 1\right), \\
& b_{n m}^{(1)}=- \kappa \frac{\Gamma^{2}(\tilde{\Delta}+n) \Gamma^{2}(\tilde{\Delta}+n+m)}{\Gamma(2 \tilde{\Delta}+2 n+m)} \\
& \cdot\left\{\frac{\Gamma\left(\tilde{\Delta}-\frac{1}{2} \Delta\right) \Gamma\left(\tilde{\Delta}+\frac{1}{2} \Delta-\frac{1}{2} d\right)(n) !}{\Gamma\left(2 \tilde{\Delta}-\frac{1}{2} d\right)\left(\tilde{\Delta}-\frac{1}{2} \Delta\right)_{n+1}\left(\tilde{\Delta}+\frac{1}{2} \Delta-\frac{1}{2} d\right)_{n+1}}\right. \\
&+\frac{\psi\left(\frac{1}{2} \Delta+\right.}{\left.+\tilde{\Delta}-\frac{1}{2} d+n+1\right)-2 \psi(\tilde{\Delta}+n)-2 \psi(\tilde{\Delta}+n+m)+2 \psi(2 \tilde{\Delta}+2 n+m)}(n+1) !\left(\frac{1}{2} \Delta+\tilde{\Delta}-\frac{1}{2} d\right) \\
& \times{ }_{3} F_{2}\left(\frac{1}{2} \Delta-\tilde{\Delta}+1, \frac{1}{2} \Delta+\tilde{\Delta}-\frac{1}{2} d+1+n, 1 ; n+2, \frac{1}{2} \Delta+\tilde{\Delta}-\frac{1}{2} d+1 ; 1\right) \\
&\left.+\frac{1}{\left(\frac{1}{2} \Delta+\tilde{\Delta}-\frac{1}{2} d\right)_{n+1}} \sum_{r=0}^{\infty} \frac{(-n)_{r}\left(1+\frac{1}{2} d-\frac{1}{2} \Delta-\tilde{\Delta}\right)_{r}}{(r !)^{2}\left(\tilde{\Delta}-\frac{1}{2} \Delta+r\right)}(n+1-r)\right\}, \\
& c_{n m}^{(1)}=\kappa \frac{\Gamma^{4}\left(\frac{1}{2} \Delta\right) \Gamma^{2}\left(\tilde{\Delta}-\frac{1}{2} \Delta\right) \Gamma^{2}\left(\tilde{\Delta}+\frac{1}{2} \Delta-\frac{1}{2} d\right)}{\Gamma(\Delta) \Gamma\left(2 \tilde{\Delta}-\frac{1}{2} d\right) \Gamma\left(\Delta-\frac{1}{2} d+1\right)} \frac{\left(\frac{1}{2} \Delta\right)_{n}^{2}\left(\frac{1}{2} \Delta\right)_{n+m}^{2}}{(\Delta)_{2 n+m}\left(\Delta-\frac{1}{2} d+1\right)_{n}} .
\end{aligned}
$$

The Pochhammer symbol $(a)_{n}$ is defined as

$$
(a)_{n}=\frac{\Gamma(a+n)}{\Gamma(a)} .
$$

The hypergeometric functions which appear in (24) and (25) can be given in term of terminating series by virtue of the identity given in (85). However, due to identities of the form

$$
\lim _{a \rightarrow 0} \frac{\psi(a-r)}{\Gamma(a-r)}=(-1)^{r+1} \Gamma(r+1)
$$


the last term in (25) gives a contribution proportional to a ${ }_{4} F_{3}$ generalized hypergeometric function. It is easy to see that (23) is analytic in the direct channel limit $u \rightarrow 0$ and $v \rightarrow 1$.

\subsection{The crossed channel}

The calculations in both the crossed channels ( $x_{3} \leftrightarrow x_{4}$ or $u \leftrightarrow v$ and $x_{3} \leftrightarrow x_{2}$ or $u \leftrightarrow 1 / u$ and $v \leftrightarrow v / u$ ), are significantly more complicated as they involve the analytic continuation of the result (23). The reason is that we want to obtain an expression which can be matched with the direct channel $\mathrm{OPE}$, therefore we require that the result be written e.g. in the general form of (6). Consider for clarity the crossed channel obtained from (21) by the interchange $u \leftrightarrow v$.

This can be achieved in two fashions. We start first from the MellinBranes integral for $B\left(x_{1}, x_{4} ; x_{2}, x_{3}\right)$ (say) analogous to (21), and expand it into contributions of the poles in $s$ to the right of the integral contour. We obtain a decomposition into a contribution from $\mathcal{O}_{\sigma}$ exchange and from the exchange of an infinite tower of the tensor fields. Because of the missing shadow terms (see Sections 4.1, 4.2) each contribution is singular at $v=1$. In Appendix $\mathrm{B}$ we show that for the specific case of the B graph in Fig. 1, the non-analytic terms cancel each other by virtue of highly non-trivial identities for the generalized hypergeometric function ${ }_{3} F_{2}$. The explicit form of the remaining analytic terms can also be derived using the generalized hypergeometric differential equation and the representations of its solutions by Mellin-Barnes integrals (Appendix B).

The second method makes use of the Mellin-Barnes integral for $B\left(x_{1}, x_{4} ; x_{2}, x_{3}\right)$ itself and we observe that (after the exchange of $u \leftrightarrow v$ in (21)) the power $v^{s}$ can be Taylor expanded at $v=1$ under the 
integral sign (Appendix B). Here we just give a general formula which is useful in extracting information for the possible general structure of the conformal OPE from AdS/CFT correspondence. Namely, the result for both the crossed channel graphs obtained from Fig.1 is of the form

$$
\begin{aligned}
& B\left(x_{1}, x_{4} ; x_{2}, x_{3}\right)+B\left(x_{1}, x_{2} ; x_{3}, x_{4}\right) \\
& =\frac{1}{\left(x_{12}^{2} x_{34}^{2}\right)^{\tilde{\Delta}}} \sum_{n, m=0}^{\infty} \frac{u^{n}(1-v)^{m}}{n ! m !}\left[-\tilde{a}_{n m} \ln u+\tilde{b}_{n m}\right]
\end{aligned}
$$

where the coefficients $\tilde{a}_{n m}, \tilde{b}_{n m}$ depend solely on $\Delta, \tilde{\Delta}$ and $d$.

\section{General scalar and tensor exchange in CFT}

In order to analyze the AdS results of Section 3 in terms of CFT partial waves, we need a formalism which allows the identification of a general conformal tensor and all its derivatives in the four-point function (14). To achieve this we construct four-point amplitudes with covariant vertices and a general tensor field of rank $l$ and dimension $\Delta$ exchanged in the direct channel. The appropriate conformal graph is depicted in Fig.2 and gives the conformally invariant local result $\beta_{\tilde{\Delta}}\left(x_{1}, x_{2}, x_{3}, x_{4} ; \Delta, l\right)$.

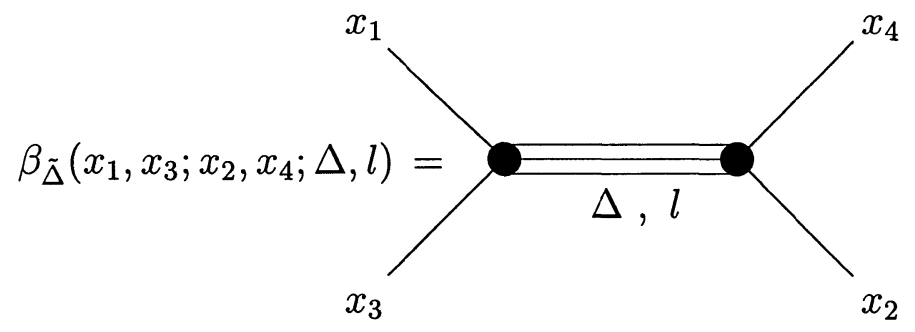

Figure 2: The tensor exchange graphs. The dark blobs correspond to the full vertex functions obtained by suitable amputation of (41). 


\subsection{Scalar exchange in CFT}

To begin with we consider the exchange of a general scalar field of dimension $\Delta$ in CFT. This corresponds to the $l=0$ graphs in Fig.2. The three-point functions (12) which appear in it are contracted with the inverse two-point function. The latter is obtained from the twopoint function (10) as

$$
\begin{aligned}
& \int \mathrm{d}^{d} z\left\langle\mathcal{O}_{\sigma}(x) \mathcal{O}_{\sigma}(z)\right\rangle\left[\left\langle\mathcal{O}_{\sigma}(z) \mathcal{O}_{\sigma}(y)\right\rangle\right]^{-1}=\delta^{d}(x-y), \\
& {\left[\left\langle\mathcal{O}_{\sigma}(x) \mathcal{O}_{\sigma}(y)\right\rangle\right]^{-1}=\frac{1}{C_{\Delta}} \frac{\alpha\left(\Delta-\frac{1}{2} d\right)}{\pi^{d} \alpha(\Delta)} \frac{1}{(z-y)^{2(d-\Delta)}}} \\
& \alpha(b)=\frac{\Gamma\left(\frac{1}{2} d-b\right)}{\Gamma(b)}
\end{aligned}
$$

Then, we can use the D'EPP formula [25]

$$
\begin{aligned}
& \int \mathrm{d}^{d} x \frac{1}{\left(x_{1}-x\right)^{2 a_{1}}\left(x_{2}-x\right)^{2 a_{2}}\left(x_{3}-x\right)^{2 a_{3}}} \\
& =\frac{U\left(a_{1}, a_{2}, a_{3}\right)}{\left(x_{12}^{2}\right)^{\frac{1}{2} d-a_{3}}\left(x_{13}^{2}\right)^{\frac{1}{2} d-a_{2}}\left(x_{23}^{2}\right)^{\frac{1}{2} d-a_{1}}}, \\
& U\left(a_{1}, a_{2}, a_{3}\right)=\pi^{\frac{1}{2} d} \alpha\left(a_{1}\right) \alpha\left(a_{2}\right) \alpha\left(a_{3}\right)
\end{aligned}
$$

which is valid only for $a_{1}+a_{2}+a_{3}=d$, to obtain for the scalar exchange graph

$$
\begin{aligned}
& \beta_{\tilde{\Delta}}\left(x_{1}, x_{3}, x_{2}, x_{4} ; \Delta\right) \\
& =\frac{\left(\gamma_{*} g_{\tilde{\Delta} \tilde{\Delta} \Delta}\right)^{2}}{C_{\tilde{\Delta}}} \frac{\Gamma(\Delta) \Gamma^{2}\left(\frac{1}{2} d-\frac{1}{2} \Delta\right)}{\pi^{\frac{1}{2} d} \Gamma\left(\frac{1}{2} d-\Delta\right) \Gamma^{2}\left(\frac{1}{2} \Delta\right)} \frac{1}{\left(x_{13}^{2}\right)^{\tilde{\Delta}-\frac{1}{2} \Delta}\left(x_{24}^{2}\right)^{\tilde{\Delta}+\frac{1}{2} \Delta-\frac{1}{2} d}} \\
& \quad \times \int \mathrm{d}^{d} x \frac{1}{\left[\left(x_{1}-x\right)^{2}\left(x_{3}-x\right)^{2}\right]^{\frac{1}{2} \Delta}\left[\left(x_{2}-x\right)^{2}\left(x_{4}-x\right)^{2}\right]^{\frac{1}{2} d-\frac{1}{2} \Delta}} .
\end{aligned}
$$

The integral in (34) falls into the class of conformal 4-star integrals $[11,12,13]$ which can be evaluated using Symanzik's technique [22] due 
to the uniqueness condition satisfied by the massless scalar propagators involved in it i.e., the sum of the dimensions of the propagators is $d$. The result of the final integration is $[11,13]$

$$
\begin{aligned}
& \beta_{\tilde{\Delta}}\left(x_{1}, x_{3} ; x_{2}, x_{4} ; \Delta\right) \\
& =\frac{b u^{-\tilde{\Delta}}}{\left(x_{12}^{2} x_{34}^{2}\right)^{\tilde{\Delta}}} \sum_{n, m=0}^{\infty} \frac{u^{n}(1-v)^{m}}{n ! m !}\left[u^{\frac{1}{2} \Delta} c_{n m}(\Delta)+u^{\frac{1}{2} d-\frac{1}{2} \Delta} c_{n m}(d-\Delta)\right] \\
& c_{n m}(\Delta)=\alpha(\Delta) \alpha^{2}\left(\frac{1}{2} d-\frac{1}{2} \Delta\right) \frac{\left(\frac{1}{2} \Delta\right)_{n}^{2}\left(\frac{1}{2} \Delta\right)_{n+m}^{2}}{(\Delta)_{2 n+m}\left(\Delta-\frac{1}{2} d+1\right)_{n}} \\
& b=\frac{\left(\gamma_{*} g_{\tilde{\Delta} \tilde{\Delta} \Delta}\right)^{2}}{C_{\Delta}} \frac{\alpha^{2}\left(\frac{1}{2} \Delta\right)}{\Delta \not \alpha(}
\end{aligned}
$$

The first term on the r.h.s. of (35) is the full contribution of a scalar field with dimension $\Delta$. Its form is fully determined by conformal invariance $[26,6,7,11,13]$ and involves an infinite number of descendants of the relevant scalar field. One important observation here is that the second term in (35) is obtained from the first by the replacement

$$
\Delta \rightarrow d-\Delta .
$$

This second infinite series in (35) represents the so-called shadow symmetric singularities of the first series. ${ }^{4}$ The appearance of the shadow singularities is necessary for the cancellation of the non-analytic terms in the crossed channel of standard CFT exchange graphs [24]. The absence of shadow singularities in AdS calculations was some kind of a puzzle and its solution was proposed in the use of irregular boundary conditions $[28,29]$. The correct solution, however, is simple and physically interesting. The holographic image of AdS supergravity and all

\footnotetext{
${ }^{4}$ The term shadow symmetry was introduced for the first time in [26]. It corresponds to an intertwiner [27] of the conformal group in $d>2$ that maps the equivalent representations with dimensions $\eta$ and $d-\eta$ onto each other. Shadow symmetric singularities may correspond to physical shadow fields if the dimensions of the latter satisfy the unitarity bound e.g. $d-\eta \geq d / 2-1$. See $[11,12,13]$ and also [28].
} 
the fields which can be produced from it by operator product expansions are gauge invariant composite fields (synonymously: conformal normal products) of a set of basic fields, which we guess to be the vector supermultiplet of SYM field theory. These basic fields are not contained in the holographic image. On the other hand composite fields appear only in operator pruduct expansions which are convergent power series with increasing powers of the small distance. Shadow terms would therefore lead to a series with decreasing powers and would make the whole series twosided. There would not be a maximal small distance singularity. For more details see [30].

\subsection{Tensor exchange in CFT}

The exchange of traceless symmetric tensors of dimension $\Delta$ and rank $l$, corresponding to irreducible representations of dimension $\Delta$ and spin $l$ of $S O(d, 2)$, can be also calculated in CFT as the relevant graphs reduce to sums of scalar exchanges. For this we need to know the general expression for the conformally invariant three-point function of a symmetric, traceless tensor with dimension $\Delta$ and rank $l$ with two scalar field of dimension $\tilde{\Delta}$. This is determined from conformal invariance up to an overall constant $g_{\tilde{\Delta}, \tilde{\Delta}, \Delta, l}[6,31]$. We use the vectors

$$
\begin{aligned}
\xi_{\mu}(1,2 ; 3) & =\frac{\left(x_{13}\right)_{\mu}}{x_{13}^{2}}-\frac{\left(x_{23}\right)_{\mu}}{x_{23}^{2}} \\
\xi^{2}(1,2 ; 3) & =\frac{x_{12}^{2}}{x_{13}^{2} x_{23}^{2}}
\end{aligned}
$$


to express the three-point function as $[7,6]$

$$
\begin{aligned}
& \left\langle\mathcal{O}\left(x_{1}\right) \mathcal{O}\left(x_{3}\right) M_{\mu_{1}, \mu_{2}, \ldots, \mu_{l}}\left(x_{5}\right)\right\rangle \\
& =\frac{g_{\tilde{\Delta} \tilde{\Delta} \Delta, l} \mathcal{N}(\tilde{\Delta} ; \Delta, l)}{\left(x_{13}^{2}\right)^{\tilde{\Delta}-\frac{1}{2} \Delta}\left(x_{15}^{2} x_{35}^{2}\right)^{\frac{1}{2} \Delta}}\left[\frac{\xi_{\mu_{1}} \xi_{\mu_{2}} \cdots \xi_{\mu_{l}}}{\left(\xi^{2}\right)^{\frac{1}{2} l}}-\text { trace terms }\right] \\
& \mathcal{N}(\tilde{\Delta} ; \Delta, l)=\frac{2^{\tilde{\Delta}+\frac{1}{2} \Delta+\frac{1}{2} l}}{(2 \pi)^{\frac{1}{2} d}} \\
& \quad \cdot\left(\frac{\Gamma\left(\tilde{\Delta}+\frac{1}{2} \Delta+\frac{1}{2} l-\frac{1}{2} d\right) \Gamma\left(\tilde{\Delta}-\frac{1}{2} \Delta+\frac{1}{2} l\right) \Gamma^{2}\left(\frac{1}{2} \Delta+\frac{1}{2} l\right)}{\Gamma\left(d-\tilde{\Delta}-\frac{1}{2} \Delta+\frac{1}{2} l\right) \Gamma\left(\frac{1}{2} d-\tilde{\Delta}+\frac{1}{2} \Delta+\frac{1}{2} l\right) \Gamma^{2}\left(\frac{1}{2} d-\frac{1}{2} \Delta+\frac{1}{2} l\right)}\right)^{\frac{1}{2}}
\end{aligned}
$$

where $\xi_{\mu} \equiv \xi_{\mu}(1,3 ; 5)$. Then, the general amplitude depicted in Fig.2 consists of two covariant vertex functions one of which is amputated and can be written as

$$
\begin{aligned}
\beta_{\tilde{\Delta}}\left(x_{1}, x_{2}, x_{3}, x_{4} ; \Delta, l\right)= & \int \mathrm{d}^{d} x_{\tilde{5}}\left[\sum_{\mu_{1}, \ldots, \mu_{l}}\left\langle\mathcal{O}\left(x_{1}\right) \mathcal{O}\left(x_{3}\right) M_{\mu_{1}, \mu_{2}, \ldots, \mu_{l}}\left(x_{\tilde{5}}\right)\right\rangle\right. \\
& \left.\left\langle M_{\mu_{1}, \mu_{2}, \ldots, \mu_{l}}\left(x_{\tilde{5}}\right) \mathcal{O}\left(x_{2}\right) \mathcal{O}\left(x_{4}\right)\right\rangle_{\mathrm{amp}}\right]
\end{aligned}
$$

The amputation of the second vertex in (43) is done on the tensor field $M_{\mu_{1}, ., \mu_{l}}(x)$. With the following normalization for the two-point function $[31,6]$

$$
\begin{aligned}
& \left\langle M_{\mu_{1}, . ., \mu_{l}}\left(x_{1}\right) M_{\nu_{1}, . . \nu_{l}}\left(x_{2}\right)\right\rangle \\
& =C_{\Delta, l} \frac{\mathcal{N}(\Delta, l)}{x_{12}^{2 \Delta}}\left[\left\{I_{\mu_{1} \nu_{1}}\left(x_{12}\right) \cdots I_{\mu_{l} \nu_{l}}\left(x_{12}\right)\right\}_{s y m} \text {-traces }\right] \\
& \mathcal{N}(\Delta, l)=\frac{2^{\Delta} \Gamma(\Delta+l) \Gamma(d-\Delta-1)}{(2 \pi)^{\frac{1}{2} d} \Gamma\left(\frac{1}{2} d-\Delta\right) \Gamma(d-\Delta+l-1)} \\
& I_{\mu \nu}(x)=\delta_{\mu \nu}-2 \frac{x_{\mu} x_{\nu}}{x^{2}}
\end{aligned}
$$


the amputated vertex function appearing in (43) is obtained from (41) by the replacements

$$
\begin{aligned}
\Delta & \rightarrow d-\Delta, \\
\xi_{\mu}(1,3 ; \tilde{5}) & \rightarrow \xi_{\mu}(2,4 ; \tilde{5}) .
\end{aligned}
$$

Then from (43) we obtain

$$
\begin{aligned}
\beta_{\tilde{\Delta}}\left(x_{1}, x_{3} ; x_{2}, x_{4} ; \Delta, l\right) & \\
= & \beta_{\tilde{\Delta} ; \Delta, l} \frac{1}{\left(x_{13}^{2}\right)^{\tilde{\Delta}-\frac{1}{2} \Delta}\left(x_{24}^{2}\right)^{\tilde{\Delta}-\frac{1}{2} d+\frac{1}{2} \Delta}} \\
& \times \int \mathrm{d}^{d} x_{\tilde{5}} \frac{\left\{e_{\mu_{1}} \cdots e_{\mu_{l}}-\text { traces }\right\}\left\{e_{\mu_{1}}^{\prime} \cdots e_{\mu_{l}}^{\prime}-\text { traces }\right\}}{\left(x_{1 \tilde{5}}^{2} x_{3 \tilde{5}}^{2}\right)^{\frac{1}{2} \Delta}\left(x_{2 \tilde{5}}^{2} x_{4 \tilde{5}}^{2}\right)^{\frac{1}{2} d-\frac{1}{2} \Delta}}, \quad \\
\beta_{\tilde{\Delta} ; \Delta, l}= & \frac{g_{\tilde{\Delta} \tilde{\Delta} \Delta, l}^{2}}{C_{\Delta, l}} \frac{2^{2 \tilde{\Delta}+\frac{1}{2} d+\frac{1}{2} l} \Gamma\left(\tilde{\Delta}-\frac{1}{2} \Delta+\frac{1}{2} l\right) \Gamma\left(\tilde{\Delta}+\frac{1}{2} \Delta+\frac{1}{2} l-\frac{1}{2} d\right)}{(2 \pi)^{d} \Gamma\left(\frac{1}{2} d-\tilde{\Delta}+\frac{1}{2} \Delta+\frac{1}{2} l\right) \Gamma\left(d-\tilde{\Delta}-\frac{1}{2} \Delta+\frac{1}{2} l\right)} \\
e_{\mu}= & \frac{\xi_{\mu}(1,3 ; \tilde{5})}{\left|\xi^{2}(1,3 ; \tilde{5})\right|^{\frac{1}{2}}}, e_{\mu}^{\prime}=\frac{\xi_{\mu}(2,4 ; \tilde{5})}{\left|\xi^{2}(2,4 ; \tilde{5})\right|^{\frac{1}{2}}}, e \cdot e=e^{\prime} \cdot e^{\prime}=1 .
\end{aligned}
$$

The product of the unit vectors $e$ and $e^{\prime}$ in (43) can be evaluated in terms of Gegenbauer polynomials $C_{l}^{\frac{1}{2} d-1}(x)[23]$ as

$$
\left\{e_{\mu_{1}} \cdots e_{\mu_{l}}-\text { traces }\right\}\left\{e_{\mu_{1}}^{\prime} \cdots e_{\mu_{l}}^{\prime}-\text { traces }\right\}=\frac{1}{c_{l}^{(l)}} C_{l}^{\frac{1}{2} d-1}(t)
$$

where [12]

$$
\begin{aligned}
C_{l}^{\frac{1}{2} d-1}(t) & =\sum_{M=0}^{l} c_{l}^{(M)} t^{M} \\
t & =\frac{\xi_{\mu}(1,3 ; 5) \xi_{\mu}(2,4 ; 5)}{|\xi(1,3 ; 5)||\xi(2,4 ; 5)|} .
\end{aligned}
$$


This is derived by observing that the following generating function for Gegenbauer polynomials

$$
\left(1-2 a(\xi \cdot \eta)+a^{2} \xi^{2} \eta^{2}\right)^{1-\frac{1}{2} d} \quad \xi, \eta \in \mathbf{R}^{d},
$$

is harmonic with respect to both Laplacians $\Delta_{\xi}$ and $\Delta_{\eta}$. The latter property is equivalent to the tracelessness of the tensors in (51). Note that in (52) $c_{M}^{(l)}=0$ if $l-M$ is odd. The argument $t$ of the Gegenbauer polynomials in (52) can be expanded in powers of squares $x_{i j}^{2}$ as

$$
\begin{aligned}
& t^{M}=\left[\frac{x_{1 \tilde{5}}^{2} x_{2 \tilde{5}}^{2} x_{3 \tilde{5}}^{2} x_{4 \tilde{5}}^{2}}{4 x_{13}^{2} x_{24}^{2}}\right]^{\frac{1}{2} M} \\
& \cdot \sum_{n_{i, i+1} \in \mathbf{N}_{0}}(-1)^{n_{12}+n_{34}}\left(\begin{array}{c}
M \\
n_{12} n_{23} n_{34} n_{41}
\end{array}\right) \prod_{i=1}^{4}\left(\frac{x_{i, i+1}^{2}}{x_{i \tilde{5}}^{2} x_{i+1, \tilde{5}}^{2}}\right)^{n_{i, i+1}},
\end{aligned}
$$

with $n_{41}=n_{45}$ and $x_{5 \tilde{5}}=x_{1 \tilde{5}}$. Then, the integration of (43) can be reduced to a finite sum of four-star functions which can be evaluated to

$$
\begin{aligned}
& \beta_{\tilde{\Delta}}\left(x_{1}, x_{3}, x_{2}, x_{4} ; \Delta, l\right)=\beta_{\tilde{\Delta} ; \Delta, l} \frac{u^{-\tilde{\Delta}}}{\left(x_{12}^{2} x_{34}^{2}\right)^{\tilde{\Delta}}} \sum_{n, m=0}^{\infty} \frac{u^{n}(1-v)^{m}}{n ! m !} \\
& \times\left\{\sum_{M=0}^{l} \frac{c_{l}^{(M)}}{2^{M} c_{l}^{(l)}} \sum_{n_{i, i+1} \in \mathbf{N}_{0}}(-1)^{n_{12}+n_{34}}\left(\begin{array}{c}
M \\
n_{12} n_{23} n_{34} n_{41}
\end{array}\right) v^{n_{23}}\right. \\
& \times\left[u^{\frac{1}{2}(\Delta-M)} \alpha\left(\delta_{2}\right) \alpha\left(\delta_{4}\right) \alpha(\Delta) \frac{\left(\delta_{1}\right)_{n}\left(\frac{1}{2} d-\delta_{2}\right)_{n}\left(\delta_{3}\right)_{n+m}\left(\frac{1}{2} d-\delta_{4}\right)_{n+m}}{(\Delta)_{2 n+m}\left(\tilde{\Delta}-\frac{1}{2} d+1\right)_{n}}\right. \\
& \quad+\text { shadow term }]\} .
\end{aligned}
$$

where

$$
\begin{aligned}
& i \in(1,3) \quad \delta_{i}=\frac{1}{2}(\Delta-M)+n_{i-1, i}+n_{i, i+1}, \\
& i \in(2,4) \quad \delta_{i}=\frac{1}{2} d-\frac{1}{2}(\Delta+M)+n_{i-1, i}+n_{i, i+1} .
\end{aligned}
$$


Performing the final summations we can bring the result into the form

$$
\begin{aligned}
& \beta_{\tilde{\Delta}}\left(x_{1}, x_{3}, x_{2}, x_{4} ; \Delta, l\right) \\
& =\beta_{\tilde{\Delta} ; \Delta, l} \frac{u^{-\tilde{\Delta}}}{\left(x_{12}^{2} x_{34}^{2}\right)^{\tilde{\Delta}}} \sum_{m, n=0}^{\infty} \frac{u^{n}(1-v)^{m}}{n ! m !}\left[u^{\frac{1}{2}(\Delta-l)} D_{n m}(\Delta, l)\right. \\
& \left.+u^{\frac{1}{2}(d-\Delta-l)} D_{n m}(d-\Delta, l)\right] .
\end{aligned}
$$

The coefficients $D_{n m}$ are regular functions of $\tilde{\Delta}, \Delta$ and $d$, however their form is quite complicated and we do not present it here [12]. The first term on the r.h.s. of (59) is the full contribution of the symmetric traceless tensor field with dimension $\Delta$ and rank $l$. This is the relevant term which we need in the direct channel OPE. The second term corresponds again to the shadow symmetric singularities and it is in general absent in the AdS calculations.

\section{The structure of the OPE in the bound- ary CFT}

We are now in a position to connect the AdS results of Section 3 with the CPWA of Section 4. From the results of Section 3 we conclude that an AdS four-point amplitude $\mathcal{G}$ (i.e., an appropriately chosen set of AdS graphs), has the general form

$$
\begin{aligned}
\mathcal{G} & \left(x_{1}, x_{2}, x_{3}, x_{4}\right) \\
& =\frac{1}{\left(x_{12}^{2} x_{34}^{2}\right)^{\tilde{\Delta}}} \sum_{n, m=0}^{\infty} \frac{u^{n}(1-v)^{m}}{n ! m !}\left[u^{\frac{1}{2} \Delta-\tilde{\Delta}} c_{n m}-\mathcal{A}_{n m} \ln u+\mathcal{B}_{n m}\right], \\
\mathcal{A}_{n m} & =\left(a_{n m}^{(1)}+\tilde{a}_{n m}\right) \gamma_{*}^{2} \sim O\left(\gamma_{*}^{2}\right), \\
\mathcal{B}_{n m} & =C_{\tilde{\Delta}}^{2}\left(\delta_{m 0}+(\tilde{\Delta})_{m}\right) \delta_{0 n}+\left(b_{n m}^{(1)}+\tilde{b}_{n m}\right) \gamma_{*}^{2} \sim O(1) .
\end{aligned}
$$

The first term on the r.h.s. of (60) is the full contribution of the scalar field $\sigma(x)$ with dimension $\Delta$. This is the corresponding field of the 
scalar $\sigma(\hat{x})$ which appears in the AdS cubic vertex on (8). As a check we can calculate from (35) and (26) the coupling $g_{\tilde{\Delta} \tilde{\Delta} \Delta}$ and we find it in agreement with the AdS result (12). We conclude that this is a generic feature of the OPE in the boundary CFT obtained form AdS/CFT correspondence; the CFT scalar fields corresponding to the AdS scalars involved in the triple bulk vertices, appear intact in the operator algebra of the boundary CFT. ${ }^{5}$ We expect that this feature holds true for general tensor fields which are involved in AdS cubic vertices, however the general proof is still missing. Such a structure of the boundary OPE is robust against the inclusion of quartic couplings, since it is trivial to show that general AdS "star-graphs" give contributions of the form (29). Our results are consistent with the anticipated nonrenormalization $[19,32,18]$ of all operators in the boundary CFT which correspond to the Kaluza-Klein modes of the bulk supergravity theory.

Next we turn to the remaining two terms on the r.h.s. of (60). Following earlier works of one of the authors $[11,12]$, these terms can be matched to a conformally invariant OPE in the direct channel which includes contributions from infinite towers of symmetric, traceless tensor fields with dimensions (we replace hereafter $\Delta \rightarrow \Delta_{l, t}$ and $\beta_{\tilde{\Delta} ; \Delta, l} \rightarrow \beta_{l, t}$ )

$$
\Delta_{l, t}=2 \tilde{\Delta}+l+2 t+\eta_{l, t}
$$

where $l$ is the tensor rank and $t$ is an additional quantum number called the "twist". From Bose symmetry $l \in 2 \mathbf{N}_{0}$ and then $t \in \mathbf{N}_{0}$. These fields have an "anomalous" dimension $\eta_{l, t} \sim O\left(\gamma_{*}^{2}\right)$. Inserting (63) into (59) and expanding up to $O\left(\gamma_{*}^{2}\right)$ we get by comparing the coefficients

\footnotetext{
${ }^{5}$ Note that a cubic vertex like the one in (8) can be used to calculate the fourpoint function of the scalar field $\sigma(x)$ in the boundary CFT. In this case, some connected contributions will come from "box-graphs" which are $O\left(\gamma_{*}^{4}\right)$. In principle, such graphs can be calculated using the techniques of the present work, as was done in CFT models in in $2<d<4[11,12,13]$.
} 
of $u^{n}(1-v)^{m}$ and $u^{n}(1-v)^{m} \ln u$

$$
\begin{aligned}
\sum_{l, t} \frac{n !}{(n-t) !} D_{n m}(l, t) \beta_{l, t} & =\mathcal{B}_{n m} \\
\sum_{l, t} \frac{n !}{(n-t) !} D_{n m}(l, t) \frac{1}{2} \eta_{l, t} \beta_{l, t} & =-\mathcal{A}_{n m} .
\end{aligned}
$$

To proceed we introduce the lexicographic order in the sequences of labels $(l, t)$ and $(m, n)$ as follows (say for $(m, n))$ : we define

$$
\left(m_{1}, n_{1}\right)<\left(m_{2}, n_{2}\right)
$$

if either

$$
m_{1}+2 n_{1}<m_{2}+2 n_{2}
$$

or

$$
m_{1}+2 n_{1}=m_{2}+2 n_{2} \text { with } n_{1}<n_{2} .
$$

Moreover, we consider only those equations in (64), (65) with

$$
m+2 n \in 2 \mathbf{N}_{0} \text {. }
$$

The remaining equations are then interpreted as constraints which have to be satisfied to ensure consistency of our scheme. This has been checked to high orders in the case of the $O(N)$ vector model in $2<d<4$ $[11,12]$, and in principle can also be done in the explicit in the case of $\mathrm{AdS}_{5} / \mathrm{CFT}_{4}$ correspondence using e.g. the results of $[21]^{6}$. Then we note that the relevant labels $(m, n)$ and $(l, t)$ appear in an ordered sequence as

$$
(0,0),(2,0),(0,1),(4,0),(2,1),(0,2),(6,0), \ldots .
$$

\footnotetext{
${ }^{6}$ Work in progress
} 
This sequence can be mapped into the natural numbers maintaining the order. Using then

$$
\begin{aligned}
\tilde{D}_{(m, n),(l, t)} & =\frac{n !}{(n-t) !} D_{n m}(l, t) \\
\tilde{\mathcal{B}}_{(m, n)} & =\mathcal{B}_{n m}, \tilde{\mathcal{A}}_{(m, n)}=-2 \mathcal{A}_{n m},
\end{aligned}
$$

we can write $(64),(65)$ in a matrix notation as

$$
\begin{aligned}
\sum_{s} \tilde{D}_{r s} \beta_{s} & =\tilde{\mathcal{B}}_{r} \\
\sum_{s} \tilde{D}_{r s} \beta_{s} \eta_{s} & =\tilde{\mathcal{A}}_{r}
\end{aligned}
$$

where $s$ and $r$ now denote the above pairs of indices. These equations can be solved if we notice that

$$
\tilde{D}_{r s}=0 \quad \text { if } \quad r<s
$$

i.e., $\tilde{D}_{r s}$ is a triangular matrix. Moreover we have

$$
\tilde{D}_{r r} \neq 0
$$

and this allows to write the general solution of (71) and (73) as

$$
\begin{aligned}
\beta_{s} & =\sum_{1 \leq r \leq s}\left(\tilde{D}^{-1}\right)_{s r} \tilde{B}_{r} \\
\beta_{s} \eta_{s} & =\sum_{1 \leq r \leq s}\left(\tilde{D}^{-1}\right)_{s r} \tilde{A}_{r} .
\end{aligned}
$$

The above constitute, in principle, the general solution to the problem of evaluating the couplings and anomalous dimensions of all scalar and tensor fields which appear in the OPE of the boundary CFT. These equations have been shown to work in the case of the conformally invariant $O(N)$ vector in $2<d<4$ [12]. Given the values of the dimensions $\tilde{\Delta}$ and $\Delta$, they can also be applied to any form of AdS/CFT 
correspondence. However, some technical complications might arise in explicit calculations due to our choice of the normalization of the twoand three-point functions of tensor fields (44) and (41) (see for example [35]). For specific values of the tensor dimensions and rank, it may seem that the normalization constants and coupling include divergences or zeroes. This is related e.g. to similar problems encountered in calculations of scalar extremal correlators [36]. Nevertheless, it can be easily shown [12] that in all cases the final formulae (77) and (78) yield regular results once an overall normalization is suitably chosen.

\section{Summary and Outlook}

In this work we studied a conformally invariant scalar four-point function which is obtained from $\mathrm{AdS}_{d+1} / \mathrm{CFT}_{d}$ correspondence. We calculated the AdS scalar exchange graphs both in the direct and the crossed channels and demonstrated that they give analytic results. This is necessary in order that the corresponding four-point function in the boundary CFT admits an OPE. We then presented a general procedure to obtain the contribution of scalar and tensor fields in conformally invariant four-point functions. Interpreting the logarithms coming from the AdS calculation as anomalous dimensions of scalar and tensor fields in the boundary CFT, we were then able to present a general method for their evaluation by matching the AdS results with the conformal OPE. As a by-product, we presented some highly non-trivial formulae for the analytic continuation of generalized ${ }_{3} F_{2}$ hypergeometric functions.

Our results indicate a possible general form for the OPE of the boundary CFT. Namely, it seems that AdS scalar fields which are involved in cubic vertices in the bulk, manifest themselves intact in the operator algebra of the boundary CFT. This essentially means that 
once their dimensions and couplings have been fixed from the supergravity reduction, they will not change at any order in the "Witten graph" expansion. The latter gives, according to the Maldacena conjecture [1], the strong coupling limit of the boundary CFT. Therefore, if these fields appear also in a free-field realization of the boundary CFT, we conclude that they are non-renormalized. This is in agreement with the general view $[19,32,18]$ that the Kaluza-Klein modes of the compactified supergravity, which are actually the ones appearing in the cubic AdS vertices, are not renormalized. Fields which appear either in the strong or in the free-field realization of the boundary CFT may respectively be either string modes or non-chiral primaries, the latter in the case of $\mathcal{N}=4 \mathrm{SYM}_{4}$. In this latter case, the scalar and tensor fields whose anomalous dimensions are related to the logarithms of the AdS calculation correspond to composite "multi-trace" operators of the boundary CFT. Thus is seems that the non-trivial dynamics connecting the strongly and the weakly coupled realizations of $\mathcal{N}=4$ $\mathrm{SYM}_{4}$ is essentially related to the gauge group $S U(N)$.

Our calculations were performed for general $d$ and general dimensions of the scalar and tensor fields. We expect that significant simplifications will occur in specific cases such as e.g. the $\mathrm{AdS}_{5} / \mathrm{CFT}_{4}$ correspondence leading to the $\mathcal{N}=4 S U(N) \mathrm{SYM}_{4}$. However, a technical problem related to a consistent overall normalization of scalars and tensor fields may still occur in explicit calculations [35].

There a several possibilities to use and extend our results. In the case of type IIB compactifications on $\mathrm{AdS}_{5} \times \mathrm{S}^{4}$, the full bulk action has been recently calculated up to quartic interaction terms [21]. From that one can possibly calculate the four-point functions of various chiral primary operators in $\mathcal{N}=4 \mathrm{SYM}_{4}$ and study their OPEs. This would shed new light into the strong coupling dynamics of the latter the- 
ory as one could calculate the anomalous dimensions of non-protected multi-trace operators. From such calculations one could also deduce interesting results for the energy-momentum tensor or other conserved currents of the theory. Another possible application of our result would be in the case of $\mathrm{AdS}_{4} / \mathrm{CFT}_{3}$ correspondence [37]. The calculation of anomalous dimensions in this case is of particular interest, as it may be compared with existing results for the anomalous dimensions of various operators in three-dimensional CFTs $[11,12,13]$.

One additional point which needs further investigation is that the expansion (60) may not be the whole story in a perturbatively defined theory in the following sense. In general, there exists various tensors $\left(\Delta_{1}, l_{1}\right),\left(\Delta_{2}, l_{2}\right)$ with

$$
\begin{aligned}
l_{1} & =l_{2} \\
\Delta_{1} & =\Delta_{2}+\text { perturbative correction terms }
\end{aligned}
$$

The resolution of such "almost-degeneracy" requires a sufficiently high perturbative order and the description of $n$-point functions with $n>4$. For conformal sigma models in $1 / N$ expansion a method of resolution has been developed in [12]. By use of combinatorics it is possible to determine the "maximal degrees" of "almost degeneracy" using the argument that as $N \rightarrow \infty$ we obtain a free-field theory. These maximal degrees can be really trusted only for small $l[12]$. Before the question of "almost degeneracy" is resolved, an expansion such as (60) can only give averaged anomalous dimensions.

\section{Acknowledgements}

The work of A.C.P. is supported by an Alexander-von-Humboldt Research Fellowship. 


\section{A AdS exchange graphs in the direct channel}

The starting point of our calculations is formula (21). This can be obtained from (16) either by using Symanzik's technique [22, 11, 13] or following [17]. To evaluate the Mellin-Barnes integrals we find it convenient to use the non-terminating form of Saalschutz's theorem (Eq. 4.3.4.2 of [33]) for the Saalschutzian generalized hypergeometric function ${ }_{3} F_{2}$ to obtain

$$
\begin{aligned}
& B\left(x_{1}, x_{3} ; x_{2}, x_{4}\right) \\
& =\frac{\kappa \tilde{\kappa}}{\left(x_{12}^{2} x_{34}^{2}\right)^{\tilde{\Delta}}} \int_{\mathcal{C}}\left[\frac{\mathrm{d} s}{2 \pi \mathrm{i}} \frac{\Gamma(-s) \Gamma\left(\frac{1}{2} \Delta-\tilde{\Delta}-s\right) \Gamma^{4}(\tilde{\Delta}+s) \Gamma(s+1)}{\Gamma(2 \tilde{\Delta}+2 s) \Gamma\left(\frac{1}{2} \Delta+\tilde{\Delta}-\frac{1}{2} d+1+s\right)}\right. \\
& \left.\quad \times u^{s}{ }_{2} F_{1}(\tilde{\Delta}+s, \tilde{\Delta}+s ; 2 \tilde{\Delta}+2 s ; 1-v)\right] \\
& -\frac{\kappa}{\left(x_{12}^{2} x_{34}^{2}\right)^{\tilde{\Delta}}} \int_{\mathcal{C}} \frac{\mathrm{d} s}{2 \pi \mathrm{i}}\left[\Gamma^{2}(-s) \frac{\Gamma^{4}(\tilde{\Delta}+s)}{\Gamma(2 \tilde{\Delta}+2 s)} \frac{1}{(s+1)\left(\frac{1}{2} \Delta+\tilde{\Delta}-\frac{1}{2} d\right)}\right. \\
& \times{ }_{3} F_{2}\left(\frac{1}{2} \Delta-\tilde{\Delta}+1, \frac{1}{2} \Delta+\tilde{\Delta}-\frac{1}{2} d+1+s, 1 ; s+2, \frac{1}{2} \Delta+\tilde{\Delta}-\frac{1}{2} d+1 ; 1\right) \\
& \left.\quad \times u_{2}^{s}{ }_{2} F_{1}(\tilde{\Delta}+s, \tilde{\Delta}+s ; 2 \tilde{\Delta}+2 s ; 1-v)\right]
\end{aligned}
$$

where

$$
\tilde{\kappa}=\frac{\Gamma^{2}\left(\frac{1}{2} \Delta+\tilde{\Delta}-\frac{1}{2} d\right) \Gamma\left(\tilde{\Delta}-\frac{1}{2} \Delta\right)}{\Gamma\left(\frac{1}{2} \Delta-\tilde{\Delta}+1\right) \Gamma\left(2 \tilde{\Delta}-\frac{1}{2} d\right)} .
$$

The contour in the first term on the r.h.s of (81) encloses only single poles coming from $\Gamma(-s)$ and $\Gamma\left(\frac{1}{2} \Delta-\tilde{\Delta}-s\right)$ at the points $s=n$ and $s=\frac{1}{2} \Delta-\tilde{\Delta}+n, n=0,1,2, \ldots$ respectively. After expanding the hypergeometric function ${ }_{2} F_{1}$, the result obtained form the first term on the r.h.s. of $(81)$ is a double series in the variables $u$ and $(1-v)$. 
The second term on the r.h.s. of (81) is of the general form

$$
f(u)=\int_{\mathcal{C}} \frac{\mathrm{d} s}{2 \pi \mathrm{i}} \Gamma^{2}(-s) g(s) u^{s}
$$

where the function $g(s)$ does not have poles in the right half plane. To evaluate the integral we can choose a regularization method in order to disentangle the two infinite series of coincident poles coming from $\Gamma^{2}(-s)$. For example, we can choose to shift one of the infinite series of poles by an infinitesimal parameter $\epsilon$ which will be set to zero after the evaluation of the Mellin-Barnes integral. Setting then $\Gamma^{2}(-s) \rightarrow$ $\Gamma(-s) \Gamma(-s+\epsilon)$ in $(81)$ we obtain

$$
\begin{aligned}
f(u) & =\frac{\partial}{\partial \epsilon}\left[\sum_{n=0}^{\infty} \frac{u^{n-\epsilon} g(n-\epsilon)}{\Gamma^{2}(n+1-\epsilon)}\right]_{\epsilon=0} \\
& =\sum_{n=0}^{\infty} \frac{u^{n}}{(n !)^{2}}\left[2 \psi(n+1) g(n)-g(n) \ln u-\frac{\mathrm{d}}{\mathrm{d} \xi}[g(\xi)]_{\xi=n}\right]
\end{aligned}
$$

Using then (84) we can evaluate the second term on the r.h.s. of (81). The result of the lengthy calculation is given in (24)-(26) in the text. It is amusing that we are able to write the final formulas for the coefficients $a_{n m}, b_{n m}$ and $c_{n m}$ in terms of terminating series, by virtue of the following transformation

$$
\begin{aligned}
& { }_{3} F_{2}\left(\frac{1}{2} \Delta-\tilde{\Delta}+1, \frac{1}{2} \Delta+\tilde{\Delta}-\frac{1}{2} d+1+n, 1 ; n+2, \frac{1}{2} \Delta+\tilde{\Delta}-\frac{1}{2} d+1 ; 1\right) \\
& =\frac{\Gamma(n+2) \Gamma\left(\frac{1}{2} \Delta+\tilde{\Delta}-\frac{1}{2} d+1\right)}{\left(\tilde{\Delta}-\frac{1}{2} \Delta\right) \Gamma\left(\frac{1}{2} \Delta+\tilde{\Delta}-\frac{1}{2} d+1+n\right)} \\
& \quad \times{ }_{3} F_{2}\left(1-\frac{1}{2} \Delta-\tilde{\Delta}+\frac{1}{2} d, \tilde{\Delta}-\frac{1}{2} \Delta,-n ; \tilde{\Delta}-\frac{1}{2} \Delta+1,1 ; 1\right),
\end{aligned}
$$

which is a consequence of the two-term relation Eq.(2.3.3.7) of [33]. 


\section{B AdS exchange graphs in the crossed channel}

Here we present the essential steps for the crossed channel calculation corresponding to the interchange $u \leftrightarrow v$ in (81). We first observe that the argument of the ${ }_{2} F_{1}$ hypergeometric functions becomes $(1-u)$ and in order to transform it into a series in the variable $u$ we need to use a Kummer transformation e.g. Eq. 9.131.2 of [23]. Due to the form of the ${ }_{2} F_{1}$ function involved in the transformation, we encounter poles in the r.h.s. of Eq. 9.131 .2 of [23] i.e. this is a degenerate transformation. This is a consequence of the analytic continuation implied by the above Kummer transformations. To obtain the result we may use, for example, the Mellin-Barnes representation for the ${ }_{2} F_{1}$ function and appropriately regularize the coincident poles as in (84) above. It is nevertheless simple to write

$$
\begin{aligned}
{ }_{2} & F_{1}(\tilde{\Delta}+s, \tilde{\Delta}+s ; 2 \tilde{\Delta}+2 s ; 1-u) \\
\equiv & \left.{ }_{2} F_{1}(\tilde{\Delta}+s, \tilde{\Delta}+s ; 2 \tilde{\Delta}+2 s+\epsilon ; 1-u)\right|_{\epsilon \rightarrow 0} \\
= & {\left[\frac{\Gamma(2 \tilde{\Delta}+2 s+\epsilon) \Gamma(\epsilon)}{\Gamma^{2}(\tilde{\Delta}+s+\epsilon)}{ }_{2} F_{1}(\tilde{\Delta}+s, \tilde{\Delta}+s ; 1-\epsilon ; u)\right.} \\
& \left.+u^{\epsilon} \frac{\Gamma(2 \tilde{\Delta}+2 s+\epsilon) \Gamma(-\epsilon)}{\Gamma^{2}(\tilde{\Delta}+s)}{ }_{2} F_{1}(\tilde{\Delta}+s+\epsilon, \tilde{\Delta}+s+\epsilon ; 1+\epsilon ; u)\right]_{\epsilon \rightarrow 0} \\
= & \frac{\Gamma(2 \tilde{\Delta}+2 s)}{\Gamma^{4}(\tilde{\Delta}+s)} \sum_{n=0}^{\infty} \frac{u^{n}}{(n !)^{2}} \Gamma^{2}(\tilde{\Delta}+s+n) \\
& \cdot[-\ln u+2 \psi(n+1)-2 \psi(\tilde{\Delta}+s+n)] \\
= & \left.\frac{\Gamma(2 \tilde{\Delta}+2 s)}{\Gamma^{4}(\tilde{\Delta}+s)} \sum_{n=0}^{\infty} \frac{u^{n}}{(n !)^{2}} \mathcal{D}_{n}\left(\frac{\partial}{\partial \xi}\right) \Gamma^{2}(\tilde{\Delta}+s+n+\xi)\right|_{\xi=0},
\end{aligned}
$$


where

$$
\mathcal{D}_{m}\left(\frac{\partial}{\partial \xi}\right)=-\ln u+2 \psi(m+1)-\frac{\partial}{\partial \xi} .
$$

Using then (86) we obtain from (81)

$$
\begin{aligned}
& B\left(x_{1}, x_{4} ; x_{2}, x_{3}\right) \\
& =\frac{\kappa \hat{\kappa}}{\left(x_{12}^{2} x_{34}^{2}\right)^{\tilde{\Delta}}}\left[\sum _ { m = 0 } ^ { \infty } \frac { u ^ { m } } { ( m ! ) ^ { 2 } } \mathcal { D } _ { m } ( \frac { \partial } { \partial \xi } ) \left(v^{\frac{1}{2} \Delta-\tilde{\Delta}} f_{1}(v, \xi)\right.\right. \\
& \left.\left.\quad \quad-f_{2}(v, \xi)-f_{3}(v, \xi)\right)\right]_{\xi=0} \\
& f_{1}(v, \xi) \\
& =\frac{\Gamma^{2}\left(\frac{1}{2} \Delta+m+\xi\right)}{\Gamma\left(\Delta-\frac{1}{2} d+1\right)}{ }_{2} F_{1}\left(\frac{1}{2} \Delta+m+\xi, \frac{1}{2} \Delta+m+\xi ; \Delta-\frac{1}{2} d+1 ; v\right), \\
& f_{2}(v, \xi) \\
& =\frac{\Gamma^{2}(\tilde{\Delta}+m+\xi)}{\Gamma\left(\frac{1}{2} \Delta+\tilde{\Delta}-\frac{1}{2} d+1\right) \Gamma\left(\tilde{\Delta}-\frac{1}{2} \Delta+1\right)} \\
& \quad \times{ }_{3} F_{2}\left(\tilde{\Delta}+m+\xi, \tilde{\Delta}+m+\xi, 1 ; \frac{1}{2} \Delta+\tilde{\Delta}-\frac{1}{2} d+1, \tilde{\Delta}-\frac{1}{2} \Delta+1 ; v\right), \\
& f_{3}(v, \xi) \\
& =\frac{\Gamma^{2}\left(\tilde{\Delta}-\frac{1}{2} \Delta\right) \Gamma^{2}\left(\tilde{\Delta}-\frac{1}{2} \Delta-\frac{1}{2} d\right)}{\mathcal{C}} \frac{\mathrm{d} s}{2 \pi \mathrm{i}} \frac{\Gamma^{2}(-s) \Gamma^{2}(\tilde{\Delta}+m+s+\xi)}{(s+1)\left(\tilde{\Delta}+\frac{1}{2} \Delta-\frac{1}{2} d\right)} v^{s} \\
& \times{ }_{3} F_{2}\left(\frac{1}{2} \Delta-\tilde{\Delta}+1, \frac{1}{2} \Delta+\tilde{\Delta}-\frac{1}{2} d+1+s, 1 ; s+2, \frac{1}{2} \Delta+\tilde{\Delta}-\frac{1}{2} d+1 ; 1\right),
\end{aligned}
$$

with

$$
\hat{\kappa}=\frac{\Gamma^{2}\left(\frac{1}{2} \Delta+\tilde{\Delta}-\frac{1}{2} d\right) \Gamma^{2}\left(\tilde{\Delta}-\frac{1}{2} \Delta\right)}{\Gamma\left(2 \tilde{\Delta}-\frac{1}{2} d\right)} .
$$


First we show that $f_{3}(v, \xi)$ does not contain non-analytic terms as $v \rightarrow$ 1. Using (84) we can write

$$
f_{3}(v, \xi)=\frac{\partial}{\partial \epsilon}\left[\sum_{n=0}^{\infty} \frac{v^{n-\epsilon} g(n-\epsilon, \xi)}{\Gamma^{2}(n+1-\epsilon)}\right]_{\epsilon=0} .
$$

The possible non-analytic terms as $v \rightarrow 1$ in (93) are determined by the large- $n$ asymptotics of the ratio $g(n-\epsilon, \xi) / \Gamma^{2}(n+1-\epsilon)$. This in turn can be found using the Stirling formula below [23]

$$
\begin{aligned}
\left.\frac{\Gamma(a+r+1)}{\Gamma(r+1)}\right|_{r \rightarrow \infty} & \approx \exp \left[a \ln (r+1)+\sum_{k=1}^{\infty} \frac{\mathcal{P}_{k+1}(a)}{(r+1)^{k}}\right] \\
\mathcal{P}_{k+1}(a) & =\frac{(-1)^{k+1}}{k} \sum_{l=0}^{n}\left(\begin{array}{c}
n \\
l
\end{array}\right) \frac{B_{l}}{n-l+1}(a-1)^{n-l+1}, a \in \mathbf{C} \\
& =\frac{(-1)^{k+1}}{k} \sum_{l=0}^{a-1} l^{k}, a \in \mathbf{N} .
\end{aligned}
$$

where $B_{l}$ are the Bernoulli numbers (Secs. 9.61, 9.71 of [23]). This enables us to obtain an asymptotic expansion for ${ }_{3} F_{2}$ in (91) by virtue of Eq. 4.3.4.2 of [33] setting (in Slater's notation) $c=\frac{1}{2} \Delta+\tilde{\Delta}-\frac{1}{2} d+1+s$, $e=s+2$. In this way we obtain

$$
\left.\frac{g(n-\epsilon, \xi)}{\Gamma^{2}(n+1-\epsilon)}\right|_{n \rightarrow \infty} \approx \sum_{i=1}^{2} \sum_{\lambda}^{\infty} \tilde{\sigma}_{\lambda, i} \frac{\Gamma\left(A_{i}+n+1-\epsilon-\lambda\right)}{\Gamma(n+1-\epsilon)}
$$

for some parameters $A_{i}$, which depend among others on $m$ and $\xi$. The coefficients $\tilde{\sigma}_{\lambda, i}$ can in principle be explicitly determined in terms of the Bernoulli numbers [24], but in this case we only require their existence. 
Then, by virtue of (96) we obtain the non-analytic terms

$$
\begin{aligned}
& \left.\sum_{n=0}^{\infty} \frac{v^{n-\epsilon} g(n-\epsilon, \xi)}{\Gamma^{2}(n+1-\epsilon)}\right|_{n . a .} \\
& \left.\approx \sum_{i=1}^{2} \sum_{\lambda}^{\infty} \tilde{\sigma}_{\lambda, i} v^{-\epsilon} \frac{\Gamma\left(A_{i}+1-\epsilon-\lambda\right)}{\Gamma(1-\epsilon)}{ }_{2} F_{1}\left(A_{i}+1-\epsilon-\lambda, 1 ; 1-\epsilon ; v\right)\right|_{n . a .} \\
& \approx \sum_{i=0}^{2} \sum_{\lambda}^{\infty} \tilde{\sigma}_{\lambda, i} \Gamma\left(A_{i}+1-\lambda\right)(1-v)^{-A_{i}-1+\lambda}
\end{aligned}
$$

where to get the second line of (97) we used a Kummer transformation and have omitted the analytic terms. The crucial point is now that the non-analytic terms are independent of the parameter $\epsilon$ and therefore drop out when we substitute (97) into (93).

We next consider the function

$$
v^{\frac{1}{2} \Delta-\tilde{\Delta}} f_{1}(v, \xi)-f_{2}(v, \xi)
$$

The assertion that (98) is holomorphic at $v=1$ is a mathematical theorem that will be proved first. Our presentation of the proof follows the review article of Norlund [34].

Using a Kummer transformation we can see that the first term in (98) involves the non-analytic part

$$
\begin{aligned}
& (1-v)^{1-\frac{1}{2} d-2 m-2 \xi} \Gamma\left(\frac{1}{2} d-1+2 m+2 \xi\right) \\
& \cdot \sum_{r, n=0}^{\infty} \frac{(1-v)^{r+n}}{r ! n !} \frac{\left(\tilde{\Delta}-\frac{1}{2} \Delta\right)_{r}\left(\frac{1}{2} \Delta-\frac{1}{2} d+1-m-\xi\right)_{n}^{2}}{\left(2-\frac{1}{2} d-2 m-2 \xi\right)_{n}}
\end{aligned}
$$

where we have used the binomial expansion for $v^{\frac{1}{2} \Delta-\tilde{\Delta}}$. We will show that the second term in (98) involves a non-analytic term which just cancels the above contribution. 
The second term of (98) can be brought into the form (Eq. 1.13 of [34])

$$
\begin{gathered}
\Phi_{s}(v)=v^{\gamma_{s}} \frac{\left[\prod_{r=1}^{3} \Gamma\left(\alpha_{r}+\gamma_{s}\right)\right]}{\left[\prod_{r \neq s} \Gamma\left(\gamma_{r}-\gamma_{s}+1\right)\right]_{3}} F_{2}\left(\alpha_{1}+\gamma_{s}, \alpha_{2}+\gamma_{s}, \alpha_{3}+\gamma_{s}\right. \\
\left.\gamma_{s}-\gamma_{1}+1, \gamma_{s}-\gamma_{2}+1, \gamma_{s}-\gamma_{3}+1 ; v\right)^{\dagger}
\end{gathered}
$$

where $s=1,2,3$ and the ${ }^{\dagger}$ indicates that the argument $\gamma_{s}-\gamma_{s}+1=1$ is left out. In this representation (100) satisfies the differential equation

$$
\left[\mathcal{Q}\left(v \frac{\mathrm{d}}{\mathrm{d} v}\right)-v \mathcal{R}\left(v \frac{\mathrm{d}}{\mathrm{d} v}\right)\right] f_{2}(v)=0
$$

where $\mathcal{Q}(x)$ and $\mathcal{R}(x)$ are the polynomials

$$
\mathcal{Q}(x)=\prod_{i=1}^{3}\left(x-\gamma_{i}\right) \quad, \quad \mathcal{R}(x)=\prod_{i=1}^{3}\left(x-\alpha_{i}\right) .
$$

The linear differential equation (101) has a regular singularity at $v=1$. As shown in [34] it possesses there one singular solution $\chi_{3}(v)$ and two regular solutions $\rho_{3}^{(1)}(v)$ and $\rho_{3}^{(2)}(v)$ which form a basis ("basis theorem"). It follows therefore that

$$
f_{2}(v)=\mathcal{C}_{s} \chi_{3}(v)+\sum_{i=1}^{2} \mathcal{C}_{r}^{(i)} \rho_{3}^{(i)}(v), 0<v<1
$$

The coefficients $\mathcal{C}_{s}, \mathcal{C}_{r}^{(1)}$ and $\mathcal{C}_{r}^{(2)}$ will be determined later. (103) constitutes a Kummer transformation for the generalized hypergeometric function ${ }_{3} F_{2}$.

The identification of the parameters in (100) is not unique. A convenient choice for our purposes is to set

$$
\begin{aligned}
s=3, \quad \alpha_{1}=\alpha_{2}=\tilde{\Delta}+m+\xi, \quad \alpha_{3}=1, \\
\gamma_{1}=\frac{1}{2} d-\frac{1}{2} \Delta-\tilde{\Delta}, \quad \gamma_{2}=\frac{1}{2} \Delta-\tilde{\Delta}, \quad \gamma_{3}=0 .
\end{aligned}
$$


With this choice we obtain from Eq. 1.15 of [34]

$$
\begin{aligned}
\beta_{1} & =\frac{1}{2} \Delta-\frac{1}{2} d-m-\xi, \\
\beta_{2}=\beta_{3} & =1-2 m-2 \xi-\frac{1}{2} d,
\end{aligned}
$$

and from Eq. 1.33 and 2.11 ibid.

$$
\begin{aligned}
\chi_{3}(v) & =\sum_{n=0}^{\infty} \frac{\mathcal{C}_{n, 3}^{(3)}}{\left(\beta_{3}+1\right)_{n}}(1-v)^{\beta_{3}+n} \\
\frac{\mathcal{C}_{n, 3}^{(3)}}{\left(\beta_{3}+1\right)_{n}} & =\sum_{r=0}^{n} \frac{\left(1+\frac{1}{2} \Delta-\frac{1}{2} d-m-\xi\right)_{r}^{2}\left(\tilde{\Delta}-\frac{1}{2} \Delta\right)_{n-r}}{r !(n-r) !\left(2-2 m-2 \xi-\frac{1}{2} d\right)_{r}}
\end{aligned}
$$

Therefore, from (103) we see that the singular part of $f_{2}(v, \xi)$ cancels the singular part $(99)$ of $f_{1}(v, \xi)$ if

$$
\mathcal{C}_{s}=\Gamma\left(2 m+2 \xi+\frac{1}{2} d-1\right)
$$

To show this we can use the method of "large order expansion" of the coefficients of $f_{2}(v, \xi)$ in powers of $v$. Namely, writing

$$
f_{2}(v, \xi)=\sum_{n=0}^{\infty} \frac{v^{n}}{n !} \frac{\Gamma^{2}(\tilde{\Delta}+m+\xi+n) \Gamma(n+1)}{\Gamma\left(\frac{1}{2} \Delta+\tilde{\Delta}-\frac{1}{2} d+1+n\right) \Gamma\left(\tilde{\Delta}-\frac{1}{2} \Delta+1+n\right)}
$$

we can use Stirling's formula (94) to obtain

$$
\begin{aligned}
& \left.\frac{\Gamma^{2}(\tilde{\Delta}+m+\xi+n)}{\Gamma\left(\frac{1}{2} \Delta+\tilde{\Delta}-\frac{1}{2} d+1+n\right) \Gamma\left(\tilde{\Delta}-\frac{1}{2} \Delta+1+n\right)}\right|_{n \rightarrow \infty} \\
& \approx \sum_{\lambda=0}^{\infty} \sigma_{\lambda} \frac{\Gamma(\beta-\lambda+n+1)}{\Gamma(n+1)}
\end{aligned}
$$

where $\beta$ is defined in (116). From (111) the non-analytic part of $f_{2}(v, \xi)$ 
can be found as

$$
\begin{aligned}
& \left.\sum_{n=0}^{\infty} \frac{v^{n}}{n !} \frac{\Gamma^{2}(\tilde{\Delta}+m+\xi+n) \Gamma(n+1)}{\Gamma\left(\frac{1}{2} \Delta+\tilde{\Delta}-\frac{1}{2} d+1+n\right) \Gamma\left(\tilde{\Delta}-\frac{1}{2} \Delta+1+n\right)}\right|_{n . a .} \\
& \approx \sum_{\lambda}^{\infty} \sigma_{\lambda}\left[\sum_{n=0}^{\infty} \frac{\Gamma(\beta-\lambda+n+1)}{\Gamma(n+1)} v^{n}\right] \\
& \approx \sum_{\lambda=0}^{\infty} \sigma_{\lambda} \Gamma\left(\frac{1}{2} d-1+2 m+2 \xi-\lambda\right)(1-v)^{1-\frac{1}{2} d-2 m-2 \xi+\lambda}
\end{aligned}
$$

Since from (108) and (111)

$$
\sigma_{0}=\frac{\mathcal{C}_{0,3}^{(3)}}{\left(\beta_{3}+1\right)_{0}}=1,
$$

then (109) follows.

In our previous work [24], being unaware of [34] we went on to show that the coefficients $\sigma_{\lambda}$ in (111) equal the r.h.s of (108) for all $\lambda$. The explicit formula for the $\sigma_{\lambda}$ is obtained recursively from

$$
\begin{aligned}
& \sum_{\lambda=0}^{\infty} \frac{\sigma_{\lambda}}{(n+1)^{\lambda}} \exp \left[\sum_{k=1}^{\infty} \frac{\mathcal{P}_{k+1}(\beta-\lambda)}{(n+1)^{k}}\right] \\
& \underset{n \rightarrow \infty}{\approx} \exp \left[\sum_{k=1}^{\infty} \frac{2 \mathcal{P}_{k+1}\left(t_{1}\right)-\mathcal{P}_{k+1}\left(t_{2}\right)-\mathcal{P}_{k+1}\left(t_{3}\right)}{(n+1)^{k}}\right], \\
& t_{1}=\tilde{\Delta}+m+\xi-1, t_{2}=\tilde{\Delta}-\frac{1}{2} \Delta, t_{3}=\frac{1}{2} \Delta+\tilde{\Delta}-\frac{1}{2} d, \\
& \beta=2 t_{1}-t_{2}-t_{3}=\frac{1}{2} d-2+2 m+2 \xi,
\end{aligned}
$$

by matching the powers of $1 /(n+1)$ on both sides of $(114)$. Then, from (108) and (112) we have to prove that

$$
\begin{aligned}
& \sum_{\lambda=0}^{\infty} \sigma_{\lambda} \frac{(-1)^{\lambda}(1-v)^{\lambda}}{\left(2-\frac{1}{2} d-2 m-2 \xi\right)_{\lambda}} \\
& =\sum_{k, l=0}^{\infty} \frac{(1-v)^{k+l}}{k ! l !} \frac{\left(\tilde{\Delta}-\frac{1}{2} \Delta\right)_{l}\left(\frac{1}{2} \Delta-\frac{1}{2} d+1-m-\xi\right)_{k}^{2}}{\left(2-\frac{1}{2} d-2 m-2 \xi\right)_{k}} .
\end{aligned}
$$


To prove this we will show that

$$
\sigma_{\lambda}=\sum_{k=0}^{\lambda} \frac{(-1)^{k}}{k !(\lambda-k)}\left(t_{2}\right)_{\lambda-k}\left(t_{3}-t_{1}\right)_{k}^{2}(\beta-\lambda+1)_{\lambda-k}
$$

The proof is based on the observation that (111) and (114) have dual forms. For (111) we obtain

$$
\begin{aligned}
& \frac{\Gamma^{2}\left(t_{1}+z+1\right)}{\Gamma\left(t_{2}+z+1\right) \Gamma\left(t_{3}+z+1\right)} \\
& \underset{z \rightarrow-\infty}{\approx} \frac{\sin \pi\left(t_{2}+z\right) \sin \pi\left(t_{3}+z\right) \sin \pi(\beta+z)}{\sin ^{2} \pi\left(t_{1}+z\right) \sin \pi z} \\
& \quad \times \sum_{\lambda=0}^{\infty} \sigma_{\lambda} \frac{\Gamma(\beta-\lambda+z+1)}{\Gamma(z+1)}
\end{aligned}
$$

while for (114) we have

$$
\begin{aligned}
& \sum_{\lambda=0}^{\infty} \frac{(-1)^{\lambda} \sigma_{\lambda}}{(z+1)^{\lambda}} \exp \left[\sum_{k=1}^{\infty} \frac{\mathcal{P}_{k+1}(\beta-\lambda)}{(z+1)^{k}}\right] \\
& \underset{z \rightarrow-\infty}{\approx} \frac{\sin ^{2} \pi\left(t_{1}+z\right) \sin \pi z}{\sin \pi\left(t_{2}+z\right) \sin \pi\left(t_{3}+z\right) \sin \pi(\beta+z)} \\
& \quad \times \exp \left[\sum_{k=1}^{\infty} \frac{2 \mathcal{P}_{k+1}\left(t_{1}\right)-\mathcal{P}_{k+1}\left(t_{2}\right)-\mathcal{P}_{k+1}\left(t_{3}\right)}{(z+1)^{k}}\right],
\end{aligned}
$$

with the same $\sigma_{\lambda}$. We also need the following property

$$
\mathcal{P}_{k+1}(1-t)=(-1)^{k+1} \mathcal{P}_{k+1}(t)
$$

Now we will show that (119) follows from (118). Multiplying the r.h.s. of (118) by

$$
\frac{\Gamma(\beta-\lambda+z+1)}{\Gamma(z+1)}
$$


and summing over $\lambda$ we obtain a convergent double sum for $\operatorname{Re} z \rightarrow-\infty$

$$
\begin{aligned}
& \sum_{k, l=0}^{\infty} \frac{(-1)^{k}}{k ! l !}\left(t_{2}\right)_{l}\left(t_{3}-t_{1}\right)_{k}^{2}(\beta-l-k+1)_{l} \frac{\Gamma(\beta-l-k+z+1)}{\Gamma(z+1)} \\
& =\frac{\sin \pi z}{\sin \pi(\beta+z)} \sum_{k=0}^{\infty} \frac{\Gamma(-z)}{\Gamma(k-\beta-z)} \frac{\left(t_{3}-t_{1}\right)_{k}^{2}}{k !}{ }_{2} F_{1}\left(k-\beta, t_{2} ; k-\beta-z ; 1\right) \\
& =\frac{\sin \pi z}{\sin \pi(\beta+z)} \frac{\Gamma\left(-z-t_{2}\right)}{\Gamma\left(-\beta-z-t_{2}\right)}{ }_{2} F_{1}\left(t_{3}-t_{1}, t_{3}-t_{1} ;-\beta-z-t_{2} ; 1\right) \\
& =\frac{\sin ^{2} \pi\left(t_{1}+z\right) \sin \pi z}{\sin \pi\left(t_{2}+z\right) \sin \pi\left(t_{3}+z\right) \sin \pi(\beta+z)} \frac{\Gamma^{2}\left(t_{1}+z+1\right)}{\Gamma\left(t_{2}+z+1\right) \Gamma\left(t_{3}+z+1\right)} .
\end{aligned}
$$

Since all the above steps are invertible, (117) and (118) are proven q.e.d.

Next we turn to the analytic part of $f_{2}(v, \xi)$ in (103). We can then write from Sec. 5.7 of [34]

$$
\rho_{3}^{(1)}(v)=y_{1,2}(v), \quad \rho_{3}^{(2)}(v)=y_{1,3}(v)
$$

where e.g.

$$
y_{1,2}(v)=\int_{\mathcal{C}} \frac{\mathrm{d} s}{2 \pi \mathrm{i}} v^{s} \frac{\Gamma\left(\gamma_{1}-s\right) \Gamma\left(\gamma_{2}-s\right)\left[\prod_{i=1}^{3} \Gamma\left(\alpha_{i}+s\right)\right]}{\Gamma\left(s+1-\gamma_{3}\right)} .
$$

The contour $\mathcal{C}$ separates the increasing from the decreasing sequence of poles. The integral in (125) converges for

$$
2 \pi>\arg v>-2 \pi
$$

which includes the circle

$$
|1-v| \leq 1
$$

Then, an expansion of $y_{1,2}(v)$ in powers of $(1-v)$ is given by Eq. 5.35 
of $[34]$ as

$$
\begin{aligned}
y_{1,2}(v)= & C_{1} v^{\gamma_{2}} \sum_{n=0}^{\infty} \frac{\left(\alpha_{1}+\gamma_{2}\right)_{n}\left(\alpha_{2}+\gamma_{2}\right)_{n}}{n !\left(\alpha_{1}+\alpha_{2}+\gamma_{1}+\gamma_{2}\right)_{n}}(1-v)^{n}{ }_{3} F_{2}\left(\alpha_{1}+\gamma_{1}, \alpha_{2}+\gamma_{1},\right. \\
& \left.1-\alpha_{3}-\gamma_{3} ; \gamma_{1}-\gamma_{3}+1, \alpha_{1}+\alpha_{2}+\gamma_{1}+\gamma_{2}+n ; 1\right) \\
C_{1}= & \frac{\Gamma\left(\alpha_{1}+\gamma_{2}\right) \Gamma\left(\alpha_{2}+\gamma_{2}\right)\left[\prod_{i=1}^{3} \Gamma\left(\alpha_{i}+\gamma_{1}\right)\right]}{\Gamma\left(\alpha_{1}+\alpha_{2}+\gamma_{1}+\gamma_{2}\right) \Gamma\left(\gamma_{1}-\gamma_{3}+1\right)} .
\end{aligned}
$$

The corresponding results for $\rho_{3}^{(2)}$ are obtained from above by the interchange of indices $2 \leftrightarrow 3$.

Now we return to (103). Shifting the contour in (125) to $+\infty$ we obtain

$$
\begin{aligned}
y_{1,2}(v) & =\frac{\pi}{\sin \pi\left(\gamma_{2}-\gamma_{1}\right)}\left[\Phi_{1}(v)-\Phi_{2}(v)\right] \\
y_{1,3}(v) & =\frac{\pi}{\sin \pi\left(\gamma_{3}-\gamma_{1}\right)}\left[\Phi_{1}(v)-\Phi_{3}(v)\right] .
\end{aligned}
$$

The corresponding Mellin-Barnes representation for $\chi_{3}(v)$ reads (Eq. 2.44 of [34])

$$
\chi_{3}(v)=\Gamma\left(\beta_{3}+1\right) \int_{\mathcal{C}} \frac{\mathrm{d} s}{2 \pi \mathrm{i}} v^{-s} \prod_{r=1}^{3} \frac{\Gamma\left(s+\gamma_{r}\right)}{\Gamma\left(s-\alpha_{r}+1\right)}
$$

with the same $\beta$ as in (106). Shifting the integration contour above to $-\infty$ we obtain

$$
\chi_{3}(v)=\frac{\Gamma\left(\beta_{3}+1\right)}{\pi} \sum_{i=1}^{3} \frac{\left[\prod_{s=1}^{3} \sin \pi\left(\gamma_{i}+\alpha_{s}\right)\right]}{\left[\prod_{s \neq i} \sin \pi\left(\gamma_{i}-\gamma_{s}\right)\right]} \Phi_{i}(v)
$$

The linear system (130), (131) and (133) can be inverted. For the 
evaluation of its determinant we need the identity

$$
\begin{aligned}
& \sum_{(i, j, k) \text { in cyclic order }}^{3} \sin \pi\left(\gamma_{i}-\gamma_{j}\right) \prod_{s=1}^{3} \sin \pi\left(\gamma_{k}+\alpha_{s}\right) \\
& =-\sin \pi\left(\gamma_{1}-\gamma_{2}\right) \sin \pi\left(\gamma_{2}-\gamma_{3}\right) \sin \pi\left(\gamma_{3}-\gamma_{1}\right) \sin \pi\left(\sum_{i=1}^{3}\left(\alpha_{i}+\gamma_{i}\right)\right) .
\end{aligned}
$$

Then, for the coefficients in (103) we obtain

$$
\begin{aligned}
& \mathcal{C}_{r}^{(1)}=\frac{1}{\pi} \frac{\left[\prod_{s=1}^{3} \sin \pi\left(\gamma_{2}+\alpha_{s}\right)\right]}{\sin \pi \beta_{3} \sin \pi\left(\gamma_{3}-\gamma_{2}\right)}, \\
& \mathcal{C}_{r}^{(2)}=\frac{1}{\pi} \frac{\left[\prod_{s=1}^{3} \sin \pi\left(\gamma_{3}+\alpha_{s}\right)\right]}{\sin \pi \beta_{3} \sin \pi\left(\gamma_{2}-\gamma_{3}\right)},
\end{aligned}
$$

and also (109) again. The latter is a non-trivial check for our calculations.

Next we want to derive a Mellin-Barnes integral for the coefficients

$$
-\tilde{a}_{n m} \ln u+\tilde{b}_{n m},
$$

in (29). Here we restrict the derivation only to the contribution of $B\left(x_{1}, x_{4} ; x_{2}, x_{3}\right)$. Expanding (87) in powers of $u$ we have to treat (see (21) with $u \leftrightarrow v$ )

$$
\begin{aligned}
\frac{\kappa \kappa^{\prime \prime}}{n !} \int_{C} \frac{d s}{2 \pi i} \frac{\Gamma^{2}(-s) \Gamma\left(\frac{1}{2} \Delta-\tilde{\Delta}-s\right)}{\Gamma\left(\frac{1}{2} \Delta+\tilde{\Delta}-\frac{1}{2} d-s\right)} v_{3}^{s} F_{2}\left(\frac{1}{2} \Delta+\tilde{\Delta}-\frac{1}{2} d\right. \\
\left.\frac{1}{2} \Delta+\tilde{\Delta}-\frac{1}{2} d, \frac{1}{2} \Delta-\tilde{\Delta}-s ; \Delta-\frac{1}{2} d+1, \tilde{\Delta}+\frac{1}{2} \Delta-\frac{1}{2} d-s ; 1\right) \\
\quad \times\left.\mathcal{D}_{n}\left(\frac{\partial}{\partial \xi}\right) \Gamma^{2}(\tilde{\Delta}+s+n+\xi)\right|_{\xi=0} .
\end{aligned}
$$

By the Stirling formula [23] we know that for $s= \pm i \sigma, \sigma \rightarrow \infty$

$$
\frac{\Gamma^{2}(-s) \Gamma\left(\frac{1}{2} \Delta-\tilde{\Delta}-s\right) \Gamma^{2}(\tilde{\Delta}+s+n+\xi)}{\Gamma\left(\frac{1}{2} \Delta+\tilde{\Delta}-\frac{1}{2} d-s\right)}=O\left(e^{-2 \pi \sigma}\right) .
$$


Moreover the ${ }_{3} F_{2}$-function in (138) behaves in the same limit as a power of $\sigma$

$$
\text { const. } \times \sigma^{\max \left[0,2 \tilde{\Delta}-\frac{1}{2} d-1\right]} \text {. }
$$

An appropriate three-term relation for ${ }_{3} F_{2}(1)$ ([33], eqn.(4.3.4.2) with $a=b=\frac{1}{2} \Delta+\tilde{\Delta}-\frac{1}{2} d$,

$\left.c=\frac{1}{2} \Delta-\tilde{\Delta}-s, d=\Delta-\frac{1}{2} d+1, e=\tilde{\Delta}+\frac{1}{2} \Delta-\frac{1}{2} d-s\right)$ and the Stirling formula [23] gives a complete asymptotic series expansion for this ${ }_{3} F_{2}$-function with leading term (140). Inserting then the Taylor expansion

$$
v^{s}=\sum_{m=0}^{\infty} \frac{(1-v)^{m}}{m !}(-s)_{m}
$$

into (138) we obtain as contribution to (137) the exponentially convergent Mellin-Barnes integral

$$
\begin{aligned}
\frac{\kappa \kappa^{\prime \prime}}{n !} \int_{C} \frac{d s}{2 \pi i} \frac{\Gamma^{2}(-s) \Gamma\left(\frac{1}{2} \Delta-\tilde{\Delta}-s\right)(-s)_{m}}{\Gamma\left(\frac{1}{2} \Delta+\tilde{\Delta}-\frac{1}{2} d-s\right)}{ }_{3} F_{2}\left(\frac{1}{2} \Delta+\tilde{\Delta}-\frac{1}{2} d\right. \\
\left.\frac{1}{2} \Delta+\tilde{\Delta}-\frac{1}{2} d, \frac{1}{2} \Delta-\tilde{\Delta}-s ; \Delta-\frac{1}{2} d+1, \tilde{\Delta}+\frac{1}{2} \Delta-\frac{1}{2} d-s ; 1\right) \\
\quad \times\left.\mathcal{D}_{n}\left(\frac{\partial}{\partial \xi}\right) \Gamma^{2}(\tilde{\Delta}+s+n+\xi)\right|_{\xi=0}
\end{aligned}
$$

with

$$
\kappa^{\prime \prime}=\frac{\Gamma^{2}\left(\frac{1}{2} \Delta+\tilde{\Delta}-\frac{1}{2} d\right)}{\Gamma\left(\Delta-\frac{1}{2} d+1\right)}
$$

The holomorphy of the functions $y_{1,2}(v), y_{1,3}(v)$ at $v=1$ can equally be derived from the exponential convergence of the Mellin-Barnes integrals (125). 


\section{References}

[1] J. Maldacena, The large $N$ limit of superconformal field theories and supergravity, Adv. Theor. Math. Phys., 2 (1998), 231-252; hep-th/9711200.

S.S. Gubser, I.R. Klebanov, and A. M. Polyakov, Gauge theory correlators from noncritical string theory, Phys. Lett., B428 (1998), 105; hep-th/9802109.

E. Witten, Anti-de Sitter space and holography, Adv. Theor. Math. Phys., 2 (1998), 253-291; hep-th/9802150.

[2] 0. Aharony, S.S. Gubser, J. Maldacena, H. Ooguri, and Y. Oz, Large $N$ field theories, string theory and gravity, hep-th/9905111.

[3] A.M. Polyakov, The wall of the cave, Int. J. Mod. Phys., A14 (1999), 645; hep-th/9809057.

[4] R. Haag, Local quantum field theory, Springer (1992).

[5] K.-H. Rehren, Algebraic Holography, hep-th/9905179.

[6] E.S. Fradkin and M.Ya. Palchik, New developments in $d$ dimensional conformal quantum field theory, Phys. Rep., 300 (1998), 1.

[7] G. Mack and I.T. Todorov, Phys. Rev., D8 (1973), 1764 .

V.K. Dobrev, V.B. Petkova, S.G. Petrova, and I.T. Todorov, Phys. Rev., D13 (1976), 887.

[8] W. Mück and K.S. Viswanathan, Conformal field theory correlators from classical scalar field theory on $A d S_{d+1}$, Phys. Rev., 
D58 (1998), 041901, hep-th/9804035; Conformal field theory correlators from classical field theory on Anti-de-Sitter space II: vector and spinor fields, Phys. Rev., D58 (1998), 106006, hep-th/9805145.

[9] D.Z. Freedman, S.D. Mathur, A. Matusis, and L. Rastelli, Correlation functions in the $C F T(d) / A d S(d+1)$ correspondence, Nucl. Phys., B546 (1999), 96, hep-th/9804058.

[10] K. Symanzik, Commun. Math. Phys., 23 (1971), 49.

[11] K. Lang and W. Rühl, Nucl.Phys., B377 (1992), 371.

[12] K. Lang and W. Rühl, Nucl. Phys., B400 (1993), 597; Nucl. Phys., B402 (1993), 573.

[13] A.C. Petkou, Conserved currents, consistency relations and operator product expansions in the conformally invariant $O(N)$ vector model for $2<d<4$, Ann. Phys., 249 (1996), 180, hep-th/9419093; Operator product expansions and consistency relations in a $O(N)$ invariant fermionic $C F T$ for $2<d<4$, Phys. Lett., B389 (1996), 18, hep-th/9692954.

[14] H. Osborn and A.C. Petkou, Implications of conformal invariance for field theories in general dimensions, Ann. Phys., 231 (1994), 311, hep-th/9307010.

[15] E. D'Hoker, S.D. Mathur, A. Matusis, and L. Rastelli, The operator product expansion of $N=4 S Y M$ and the 4-point functions of supergravity, hep-th/9911222.

[16] D.Z. Freedman, S.D. Mathur, A. Matusis, and L. Rastelli, Comments on 4-point functions in the CFT/AdS correspondence, Phys. Lett., B452 (1999), 
61, hep-th/9808006.

E. D'Hoker and D. Z. Freedman, General scalar exchange in $A d S_{d+1}$, Nucl. Phys., B550 (1999), 261, hep-th/9811257.

E. D'Hoker, D.Z. Freedman, S.D. Mathur, A. Matusis, L. Rastelli, Graviton and gauge boson propagators in $A d S_{d+1}$, Nucl. Phys., B562 (1999), 330, hep-th/9902042.

E. D'Hoker, D.Z. Freedman, S.D. Mathur, A. Matusis, and L. Rastelli, Graviton exchange and complete 4-point functions in the AdS/CFT correspondence, Nucl. Phys., B562 (1999), 353, hep-th/9903196.

E. D'Hoker, D.Z. Freedman, and L. Rastelli, AdS/CFT 4-point functions: How to succeed at z-integrals without really trying, Nucl. Phys., B562 (1999), 395, hep-th/9905049.

[17] H. Liu, Scattering in Anti-de Sitter space and operator product expansion, Phys. Rev., D60 (1999), 106005, hep-th/9811152.

[18] A.C. Petkou and K. Skenderis, A non-renormalization theorem for conformal anomalies, Nucl. Phys., B561 (1999), 100 , hep-th/9906030.

[19] S. Lee, S. Minwalla, M. Rangamani, and N. Seiberg, Three-point functions of chiral primary operators in $d=4, \mathcal{N}=4$ SYM and large $N$, Adv. Theor. math. Phys., 2 (1998), 697, hep-th/9806074.

[20] G. Arutyunov and S. Frolov, Some cubic coupling in type IIB supergravity on $A d S_{4} \times S^{4}$ and three-point functions in $S Y M_{4}$ for large $N$, hep-th/9907085. 
[21] G. Arutyunov and S. Frolov, Scalar quartic couplings in type IIB supergravity on $A d S_{5} \times S^{5}$, hep-th/9912210.

[22] K. Symanzik, Lett. Nuovo Cim., 3 (1972), 734.

[23] I.S. Gradsteyn and I.M. Ryshik, Table of Integrals, Series and Products, 5th Ed. Academic Press (1994).

[24] L. Hoffmann, A.C. Petkou and W. Rühl, A note on the analyticity of AdS scalar exchange graphs in the crossed channel, hep-th/0002025.

[25] M. D’Eramo, G. Parisi, and L. Peliti, Lett. Nuovo Cim., 2 (1971), 878.

[26] S. Ferrara, A.F. Grillo, and G. Parisi, Lett. Nuovo Cim., 5 (1972), 147.

S. Ferrara, A.F. Grillo, R. Gatto, and G. Parisi, Lett. Nuovo Cim., 4 (1972), 115.

S. Ferrara and G. Parisi, Nucl. Phys., B42 (1972), 281.

[27] K. Koller, Commun. Math. Phys., 40 (1975), 15.

[28] I. Klebanov and E. Witten, AdS/CFT correspondence and symmetry breaking, Nucl. Phys., B556 (1999), 89, hep-th/9905104.

[29] W. Mück and K.S. Viswanathan, Regular and irregular boundary conditions in the AdS/CFT correspondence, Phys. Rev., D60 (1999), 081901, hep-th/9906155.

[30] T. Leonhard and W. Rühl, to appear. 
[31] V.K. Dobrev, G. Mack, V.B. Petkova, S.G. Petrova, and I.T. Todorov, Rep. in Math. Phys., 9 (1976) 219; Harmonic analysis on the $n$-dimensional conformal group and its applications to conformal quantum field theory, Springer-Verlag (1997).

[32] E. D'Hoker, D.Z. Freedman, and W. Skiba, Field theory tests for correlators in the AdS/CFT correspondence, Phys. Rev., D59 (1999), 045008, hep-th/9807098.

[33] L.J. Slater, Generalized Hypergeometric Functions, Cambridge University Press (1966).

[34] A.E. Norlund, Hypergeometric functions, Acta Math., 94 (1955), 289.

[35] C.P. Herzog, OPEs and 4-point functions if AdS/CFT correspondence, hep-th/0002039.

[36] E. D'Hoker, D.Z. Freedman, S.D. Mathur, A. Matusis, and L. Rastelli, Extremal correlators in the AdS/CFT correspondence, hep-th/9908160.

[37] L. Gualtieri, Harmonic analysis and superconformal gauge theories in three-dimensions from AdS/CFT correspondence, hep-th/0002116. 